\title{
Bi-layer structure of counterstreaming energetic electron fluxes: a diagnostic tool of the acceleration mechanism in the Earth's magnetotail
}

\author{
D. V. Sarafopoulos \\ Department of Electrical and Computer Engineering, Democritus University of Thrace, Xanthi, Greece \\ Received: 9 July 2008 - Revised: 7 October 2009 - Accepted: 8 December 2009 - Published: 9 February 2010
}

\begin{abstract}
For the first time we identify a bi-layer structure of energetic electron fluxes in the Earth's magnetotail and establish (using datasets mainly obtained by the Geotail Energetic Particles and Ion Composition (EPIC/ICS) instrument) that it actually provides strong evidence for a purely spatial structure. Each bi-layer event is composed of two distinct layers with counterstreaming energetic electron fluxes, parallel and antiparallel to the local ambient magnetic field lines; in particular, the tailward directed fluxes always occur in a region adjacent to the lobes. Adopting the X-line as a standard reconnection model, we determine the occurrence of bi-layer events relatively to the neutral point, in the substorm frame; four (out of the shown seven) events are observed earthward and three tailward, a result implying that four events probably occurred with the substorm's local recovery phase. We discuss the bi-layer events in terms of the $\mathrm{X}$-line model; they add more constraints for any candidate electron acceleration mechanism. It should be stressed that until this time, none proposed electron acceleration mechanism has discussed or predicted these layered structures with all their properties. Then we discuss the bi-layer events in terms of the much promising "akis model", as introduced by Sarafopoulos (2008). The akis magnetic field topology is embedded in a thinned plasma sheet and is potentially causing charge separation. We assume that as the $R_{\mathrm{c}}$ curvature radius of the magnetic field line tends to become equal to the ion gyroradius $r_{\mathrm{g}}$, then the ions become non-adiabatic. At the limit $R_{\mathrm{c}}=r_{\mathrm{g}}$ the demagnetization process is also under way and the frozen-in magnetic field condition is violated by strong wave turbulence; hence, the ion particles in this geometry are stochastically scattered. In addition, ion diffusion probably takes place across the magnetic field, since an intense pressure gradient is directed earthward; hence,
\end{abstract}

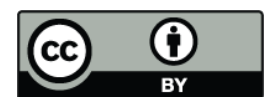

Correspondence to: D. V. Sarafopoulos (sarafo@ee.duth.gr) ions are ejected tailward of akis. This way, in front of akis an "ion capsule region" is formed with net positive charge. In between them a distinct region with an electric field $\boldsymbol{E}_{\perp}$ orthogonal to the magnetic field is emerged; $\boldsymbol{E}_{\perp}$ in front of akis is directed earthward. The field-aligned and highly anisotropic energetic electron populations have probably resulted via spatially separated antiparallel and field-aligned electric fields being the very heart of the acceleration source. We assume that the ultimate cause for the field-aligned electric fields are the net positive capsule charge and the net negative charge trapped at the tip of akis; both charges will be eventually neutralized through field aligned currents, but they remain unshielded for sufficient time to produce the observed events.

Keywords. Magnetospheric physics (Electric fields; Plasma sheet) - Space plasma physics (Charged particle motion and acceleration)

\section{Introduction}

The substorm phenomenon is extensively studied for a few decades; however, there are unresolved questions of great importance. Among the foremost of them are the energetic particle acceleration mechanisms in the Earth's magnetotail; mechanisms which are tightly associated in the past with magnetic reconnection and/or the cross-tail current disruption (Baker et al., 1979; Lopez and Baker, 1994; Lopez et al., 1989; Lui, 1996). In this work, first and foremost, we identify for the first time a bi-layer energetic electron event and establish its spatial character. We believe that it might cast light into the particle's acceleration mechanisms and the substorm's mystery at large; it is a new window to look at, that is, a new diagnostic tool to gain deeper understanding, as far as the magnetospheric dynamics is considered. We are convinced that the identified bi-layer structure

Published by Copernicus Publications on behalf of the European Geosciences Union. 
of counterstreaming energetic electron fluxes carries the fingerprint of the acceleration source. In particular, it is worthnoticing that the layer of tailward fluxes always occurs adjacent to the lobes. Certainly, it is always required that the satellite smoothly traverses the plasma sheet to capture the spatial effect.

It is commonly accepted that the spatial and temporal effects are always hard separated; nevertheless, our selected events suffice to establish the spatial character of them. Then, we are particularly interested in determining the satellite position relatively to the active source site. We begin adopting the near Earth neutral line (NENL) model (e.g., Baker et al., 1996) as the typical reconnection scheme associated with plasma flows and particle accelerations in magnetotail. Our result is that the bi-layer events are sited tailward as well as earthward relatively to the supposed X-line of the under study substorm.

The interpretation scheme of electron bi-layer events is the second major issue on which we are focused. In terms of $\mathrm{X}$-line reconnection two major questions are raised. First, that we simultaneously observe outcoming and incoming energetic electrons with respect to the active source. Second, since we observed four bi-layer events earthward and three events tailward of the supposed X-line, any X-line related electron energization mechanism should be able to produce the observed layered structures. The latter is an open subject for additional research efforts. Until this time nobody has identified observationally or predicted theoretically the studied here layered structure of counterstreaming energetic electrons.

We would like to stress that our primary intention of this work was not to contradict or to advocate the NENL model. Our basic motivation was to further scrutinize the properties of the "akis" structure, as it is introduced by Sarafopoulos (2008), and it is further elaborated here. The dynamics of akis may be the prime trigger that accelerates all the other processes in magnetotail. In this case, we will offer something related with the correct identification of processes in the cause-and-effect relationship, hence the physics. In any case, we consider that it is legitimate to try an explanation scenario which is not based on the X-line topology, but on the akis geometry with its own charge decoupling mechanism. The identification of alternate mechanisms does not diminish the $\mathrm{X}$-line models as viable possibilities. In addition, basic assumptions adopted in the standard X-type theory are also used in the akis model.

The akis model is called after the ancient Greek word $\dot{\alpha} \kappa i \varsigma$ that better expresses the geometry and functionality of the magnetic field configuration during the substorm growth phase in a thin current sheet (TCS): The stretched (tail-like) magnetic field lines locally terminate into a sharp tip (akis) around the tail midplane. Certainly, it should be underlined in advance, that our approach although potentially answers remarkable questions, however, at the same time probably throw up more fundamental questions; a process finally of- fering an opportunity for deeper understanding. Our suggestion will be extensively scrutinized-tested in the future; this author continues to look at this very promising bi-layer structure, too.

In the past, the energetic particles in general, and the energetic electron fluxes in particular, are used as the best available diagnostic tools to gain an insight into the various problems related with the large scale magnetic field topology, or the particle acceleration mechanisms and the substorm phenomenon (see the review by Krimigis and Sarris, 1980). For instance, the arrival of low energy energetic protons $(\sim 300 \mathrm{keV})$ before the higher energy ones $(\sim 1 \mathrm{MeV})$, the so-called Inverse Velocity Dispersion effect concerning short-lived energetic proton bursts inside the plasma sheet, probably reveals the growth time for the acceleration source (Sarafopoulos and Sarris, 1988). Transient and field-aligned electric fields are indirectly diagnosed on the basis of protonelectron angular distributions being appropriately modulated by them (Sarris et al., 1976; Sarafopoulos et al., 1984; Sarafopoulos and Sarris, 1987; Sarafopoulos, 2008). Most importantly, measurements concerning streaming energetic electron fluxes are to a great degree valuable; their angular distributions are not affected by any bulk plasma flow and/or any drift toward the current sheet during their time of flight from the source to satellite. In contrast, the ion fluxes frequently form energy depended layered structures in the plasma sheet; for instance, impressive case studies extended in the whole plasma sheet were studied by Sarafopoulos et al., (1997).

Energetic electron fluxes associated with substorms can be found incorporated in many papers (see for instance the works of Mitchell et al., 1990; Nagai et al., 2001; Fujimoto et al., 2001; Sarafopoulos et al., 2001; Hoshino et al., 2001; Shiokawa et al., 2003; Hoshino, 2005; Fujimoto and Machida, 2006), however, the bi-layer energetic electron event as a structure worthy of our attention is just introduced in this work.

The field-aligned and highly anisotropic electron fluxes essentially stay on field lines that directly map to their production source. The observed layered structure, therefore, probably provides an evidence for a spatial domain of antiparallel field-aligned electric fields in the very heart of the acceleration source. Hence, this structure seems to set constraints on the acceleration mechanism itself, and it has led us to assume the formation of spatially (radially) separated charges in the plasma sheet. The latter might be achieved by the akis structure embedded in a locally thinned plasma sheet: Hot ions suffer stochastic scattering in the akis region and diffuse tailward of it across the magnetic field. This way the "ion capsule" region is formed. Finally, the negatively charged akis and the positively charged capsule region may produce the observed antiparallel electron fluxes. Certainly this topology with its own dynamics will be further elaborated and discussed later on, in the appropriate place; among other issues, a critical one may concern the neutralization process which 
in effect are prevented or delayed for a while. Finally, before we deal with our observations, it is meaningful to provide our fundamental quantitative approach concerning the akis structure itself.

The development of the magnetic field geometry toward a more taillike configuration changes the nature of particle motion in the system. The intense cross-tail current concentrated in a thin sheet results in strongly curved field lines where the field component normal to the current sheet becomes very small. When the radius of curvature of the field at the current sheet becomes comparable to the particle Larmor radii, then the particles are pitch angle scattered. The transition between adiabatic and non-adiabatic or chaotic motion is dependent on both the magnetic field geometry and particle energy. When the curvature radius $R_{\mathrm{c}}$ becomes less than $\sim 9 r_{\mathrm{g}}$ (i.e., $R_{\mathrm{c}} \leq 9 r_{\mathrm{g}}$, where $r_{\mathrm{g}}$ is the particle Larmor radius), then the particles are effectively scattered and chaotization of the particle orbits begins (Sergeev and Tsyganenko, 1982; Imhof, 1988; Büchner and Zelenyi, 1989; Sergeev et al., 1996). The critical value $R_{\mathrm{c}}=r_{\mathrm{g}}$ is considered the limit of the adiabatic region beyond which the ion motion becomes chaotic (Büchner and Zelenyi, 1987, and references therein). As a matter of fact Büchner and Zelenyi (1987) used this critical value for electrons; they suggested that the chaotization of the thermal electron population removes their stabilizing effect on the tearing mode growth. We use the same critical value $R_{\mathrm{c}}=r_{\mathrm{g}}$ determining the limit between the adiabatic and chaotic ion regimes; and the same value is assumed as the tailward boundary of akis. Certainly there are additional mechanisms that eventually force ions to be ejected tailward of akis; we look at all these issues later on in the discussion section.

The possibility that the akis structure may lead to cross tail current disruption or even reconnection is qualitatively discussed in the proposed akis model; a model which under this perspective has to be considered as another approach to the mechanisms triggering substorms (Lui et al., 2008).

In Sect. 2, we exhibit the Geotail observations made up of distinct case studies. In Sect. 3, the very promising character of the under study bi-layer structure is stressed; its occurrence site in substorm terms is discussed, and an electron acceleration scheme being at the same time a possible cross tail current disruption mechanism is finally suggested.

\section{Observational evidence}

We present a few case studies demonstrating the existence of two distinct, in parallel layers, of counterstreaming energetic electron fluxes observed during "substorm episodes" in the Earth's magnetotail. Our priority is to establish that the identified bi-layer events actually provide evidence for a spatial (not temporal) domain. Our claim is that these events are not a random outcome; conversely, they carry information for the acceleration source and future work may attribute to them a key role in the magnetotail's dynamics. In particular, we stress the fact that the electron layer adjacent to the lobes is always associated with tailward directed anisotropic fluxes; on the contrary, the electron layer deeper in plasma sheet is always characterized by earthward streaming electrons. At the brief transition a few "double-peaked" angular distributions made up of mixed populations are usually observed. In each selected case the satellite must smoothly penetrates from the lobe-like domain deeper into the plasma sheet, or it may follow an anadromous route: The slow motion allows us to observe persistent flows in each flow direction. The satellite motion is traced via the $\boldsymbol{B}_{\mathrm{x}}$ component of the magnetic field being maximum at lobes and zero at neutral sheet; an effect dictated by the plasma diamagnetism in the plasma sheet.

We use the highest time-resolution energetic electron data collected by the Geotail Energetic Particles and Ion Composition EPIC/ICS instrument (Williams et al., 1994). Especially we use the ED1 channel providing electron integral fluxes with energies greater than $38 \mathrm{keV}$. The instrument's view angle is $60^{\circ}$, that is $\pm 30^{\circ}$ around the ecliptic plane. The satellite is spinning with 3-s period and, therefore, a complete angular distribution for the electron flux is obtained every 3-s. An angular distribution is composed of 8 points being the fluxes sampled in $45^{\circ}$ sectors over the ecliptic plane XY. In parallel to the electron data, the $\boldsymbol{B}_{\mathrm{X}}$ component of the magnetic field (in nanoteslas) provided by the Geotail/MGF instrument (Kokubun et al., 1994), with 3-s or better resolution, is used. In cases the Geotail plasma data are generated by taking the moments over 12-s intervals of ion distribution functions measured by the low energy particle (LEP) instrument (Mukai et al., 1994). The layered structures have occurred during substorms, something which easily can be checked by the substorm associated distinct injections at $L=6.6$. The latter data are obtained by the energetic particle differential fluxes collected by the Los Alamos National Laboratory (LANL) satellites; the instrument designed to provide these data is the Synchronous Orbit Particle Analyzer (SOPA; see Belian et al., 1992).

Provided that our observational evidence concerning the bi-layer electron structure may offer us a powerful diagnostic tool for the electron acceleration mechanism itself and the substorm phenomenon at large, we consider that it is logical to establish it presenting seven case studies belonging to four substorms. We believe that these examples suffice to remove any suspicion of random origin, and regardless to any interpretation scheme, this structure may inaugurate a new discussion. The Geotail positions for the studied events are as follows:

1. The first and second events occurred at $(\mathrm{X}, \mathrm{Y}$, $\mathrm{Z})_{\mathrm{GSM}}=(-12,-9.2,1.1) R_{\mathrm{E}}$ on 4 September 1996 ;

2. the third and the fourth event occurred at $(-30.5,0.6$, $-2.4) R_{\mathrm{E}}$ on 29 January 1998 ; 
3. the fifth event occurred at $(12,-5.7,-1.6) R_{\mathrm{E}}$ on 9 March 1995, and

4. the sixth and seventh events occurred at $(-28.6,-7.3$, -4) $R_{\mathrm{E}}$ on 25 January 1998.

Certainly, it is worth noticing that all the seven events were occurred during four well-isolated substorms. The latter may be easily checked inspecting the LANL Geosynchronous satellite energetic electron and proton data. For instance, plasma injection onsets, for the above mentioned substorms, were clearly observed (not shown here) by the

- 1991-080 satellite on 4 September 1996, at 21:40 UT,

- LANL-97A satellite on 29 January 1998, at 22:30 UT,

- 1990-095 satellite on 9 March 1995, at 05:00 UT, and

- LANL-97A satellite on 25 January 1998, at 03:50 UT.

Before each substorm onset, we discern a period of few hours with extremely low geomagnetic activity; the substorm's growth phase is also recognized by the decreases associated with the energetic particle fluxes. All the seven events are presented with almost the same format which is described in the first case study.

\subsection{Event 1}

For this case study we have plotted just two traces in Fig. 1: the integral electron fluxes with energies greater than $38 \mathrm{keV}$ and the $\boldsymbol{B}_{\mathrm{x}}$ component of the magnetic field (in $\mathrm{nTs}$ ). The shown period covers an interval of 63-s, that is 21 satellite revolutions. Each revolution corresponds to an angular distribution over the ecliptic plane; data are intentionally not spin-averaged. Hence, we recognize a persistent highly anisotropic population as successive peak fluxes with 3 -s periodicity. The repetitive and successive maximum and minimum values in Fig. 1 define the forward to backward electron flux ratio. The electrons actually demonstrate a very anisotropic flow, and the most remarkable observation is that we discern a two-layer domain. The satellite smoothly moves from the interior plasma sheet with $\boldsymbol{B}_{\mathrm{x}} \cong 13 \mathrm{nT}$ toward the plasma sheet boundary layer with $\boldsymbol{B}_{\mathrm{x}} \cong 19 \mathrm{nT}$, while somewhere in between them the electron flow direction is switched from earthward to tailward. The first six peak fluxes, which are marked with red-dashed vertical lines, are characterized by earthward flows. The (red shaded) insert angular distribution, in the upper part of Fig. 1, shows sector-averaged data for 6 satellite revolutions (corresponding to the red horizontal bar). The angular distribution values are ranged from minimum to maximum in the pertaining interval. In the insert, the directions pointing Sunward and duskward are defined, while the average magnetic field direction projected over the ecliptic plane is shown, too. Therefore, we witness the field aligned and earthward directed electron fluxes that are associated with $\boldsymbol{B}_{\mathrm{x}} \cong 14 \mathrm{nT}$.
Furthermore, the next 11 peak fluxes marked with bluedashed vertical lines show electrons streaming tailward. The latter is indicatively shown via the (blue shaded) insert angular distribution, at the lower part of Fig. 1 being the overall response for 6 satellite revolutions (corresponding to the blue horizontal bar). The very brief transition from earthward to tailward fluxes is characterized by a rather double-peaked angular distribution (i.e., bi-directional flows).

\subsection{Event 2}

This event is presented with the very same format used for the preceded case; Fig. 2 is a plot for an interval of just 83-s. Each of the 11 peak electron fluxes marked with red-dashed vertical line is characterized by an earthward streaming population. The insert angular distribution, at the upper part of Fig. 2, is the overall response for the first 6 satellite revolutions (i.e., the 18 -s interval defined by the red horizontal bar). These fluxes are related with a mean value of $\boldsymbol{B}_{\mathrm{X}} \sim 17 \mathrm{nT}$. After the just mentioned 11 peak fluxes, we do not detect any streaming population until the satellite exits into lobes. Actually, around 21:45:55 UT Geotail exits into the lobe domain, as it is evident by the very low level of energetic electron fluxes, while the $\boldsymbol{B}_{\mathrm{X}}$ is increased to $\sim 20 \mathrm{nT}$. Most importantly, just before the exit three very intense peak electron fluxes are recorded by the instrument. Thus, for the period of $\sim 9$-s (as the blue horizontal bar demarcates) the electrons clearly stream tailward. The latter is visualized with the insert angular distribution at the lower part of Fig. 2. The projected vector magnetic field on the XY plane is almost sunward directed.

\subsection{Event 3}

In this case study we scrutinize an interval of just 50-s (Fig. 3) and pay special attention to the fact that the satellite performs a short excursion departing from lobes to the central plasma sheet. Initially the satellite was placed in a plasma regime characterized by $\boldsymbol{B}_{\mathrm{x}} \cong 17 \mathrm{nT}$ and decreased electron fluxes, then the satellite smoothly penetrated at $\boldsymbol{B}_{\mathrm{x}} \cong 9 \mathrm{nT}$ and, finally, it returned back close to the departure point. Given that the blue (red) dashed-vertical lines mark the peaks of tailward (earthward) streaming energetic electrons, then it is evident that a bi-layer spatial structure is observed. In agreement with all the other studied events, the layer with tailward streaming electrons is found adjacent to the lobelike domain of plasma sheet. The very low level of electron fluxes around 22:54 UT is an authentic result due to the satellite proximity to the lobes. Three indicative angular distributions for the earthward and tailward directed electron fluxes are shown in the upper insert: Each angular distribution corresponds to the interval marked by the horizontal bar. 


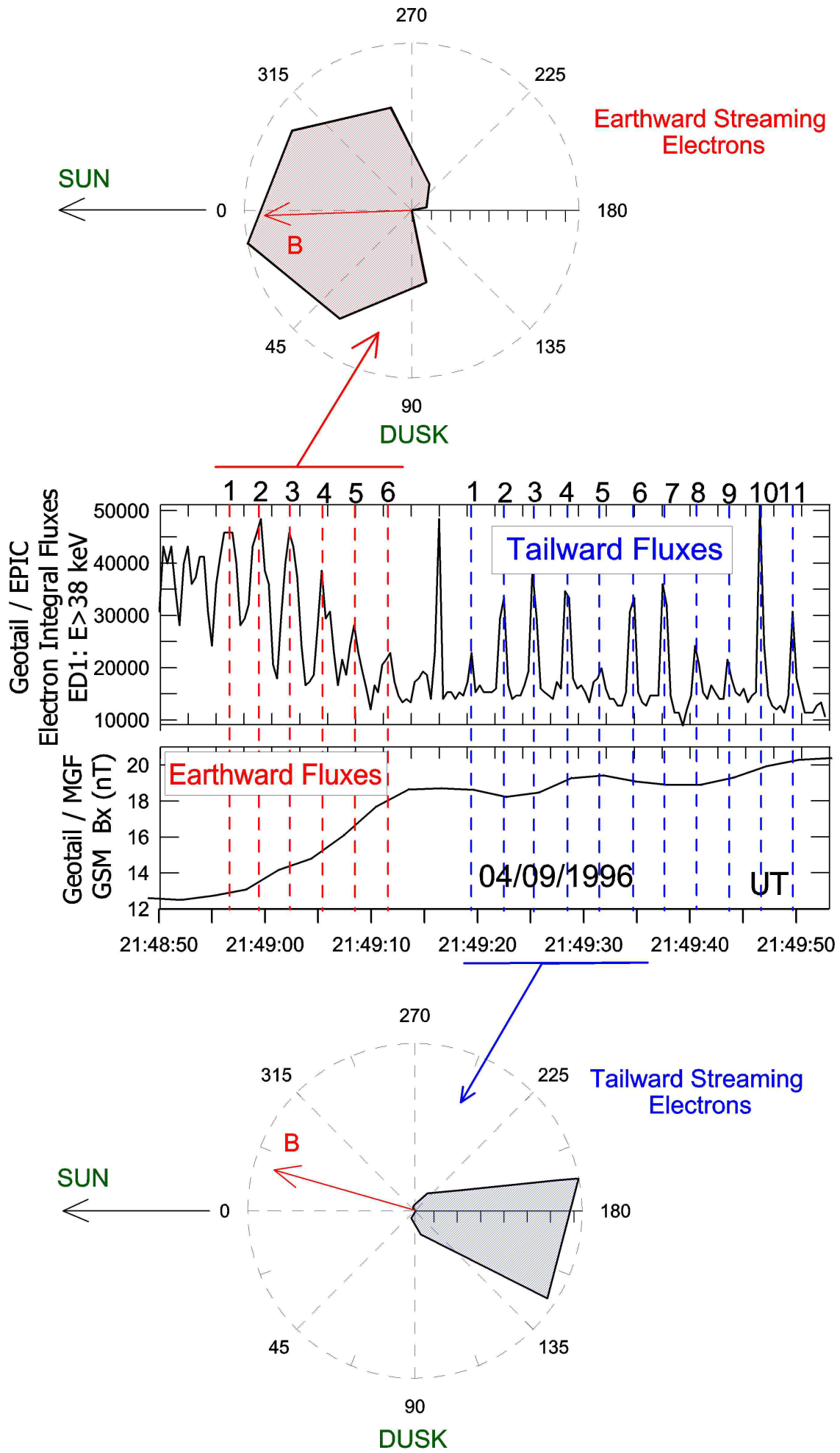

Fig. 1. Electron integral fluxes ( $E \geq 38 \mathrm{keV}$, top panel) for an interval of 63-s (corresponding to 21 Geotail revolutions) along with the $\boldsymbol{B}_{\mathrm{X}}$ component (in nTs) of the magnetic field. Fluxes are intentionally not spin-averaged: Successive peak fluxes with 3-s periodicity witness about highly anisotropic populations. The satellite smoothly moves from the interior plasma sheet with $\boldsymbol{B}_{\mathrm{X}} \cong 13 \mathrm{nT}$ toward the plasma sheet boundary layer with $\boldsymbol{B}_{\mathrm{x}} \cong 19 \mathrm{nT}$, while somewhere in between them the electron flow direction is switched from earthward (top insert) to tailward (bottom insert). The flow direction phi (in degrees) points Sunward (duskward) when $\phi=0^{\circ}\left(90^{\circ}\right)$. Each angular distribution corresponds to the interval marked with a horizontal bar. 


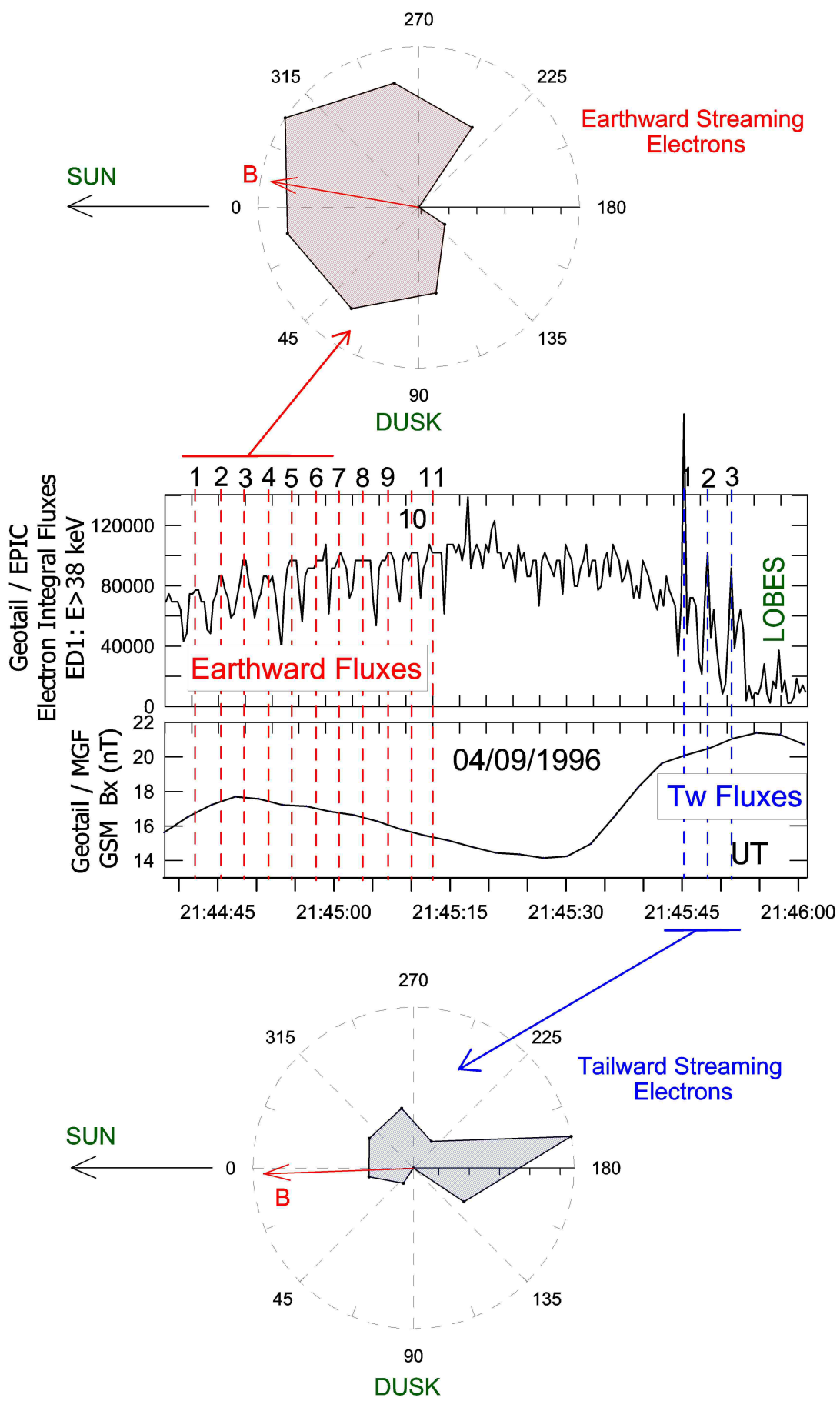

Fig. 2. Another electron bi-layer event corresponding to an interval of 83 -s; same format as in Fig. 1. The very low electron fluxes at $\sim 21: 50$ UT are associated with an exit into lobes.

\subsection{Event 4}

Again the presentation format for this event (Fig. 4) is very similar with the preceded one. We deal with an interval of 33 s (i.e., 10 satellite revolutions) showing a very smooth transition from tailward to earthward electron fluxes. Sectoraveraged data over 5 variation cycles (i.e., satellite revolu- tions), at the upper insert, underline the earthward character of flow, whereas sector-averaged data over 4 cycles at the lower insert demonstrate the tailward character of flow. Again, the switch-over in flow is interpreted as due to a spatial structure; the satellite smoothly penetrates toward the interior plasma sheet and the $\left|\boldsymbol{B}_{\mathrm{X}}\right|$ decreases. 


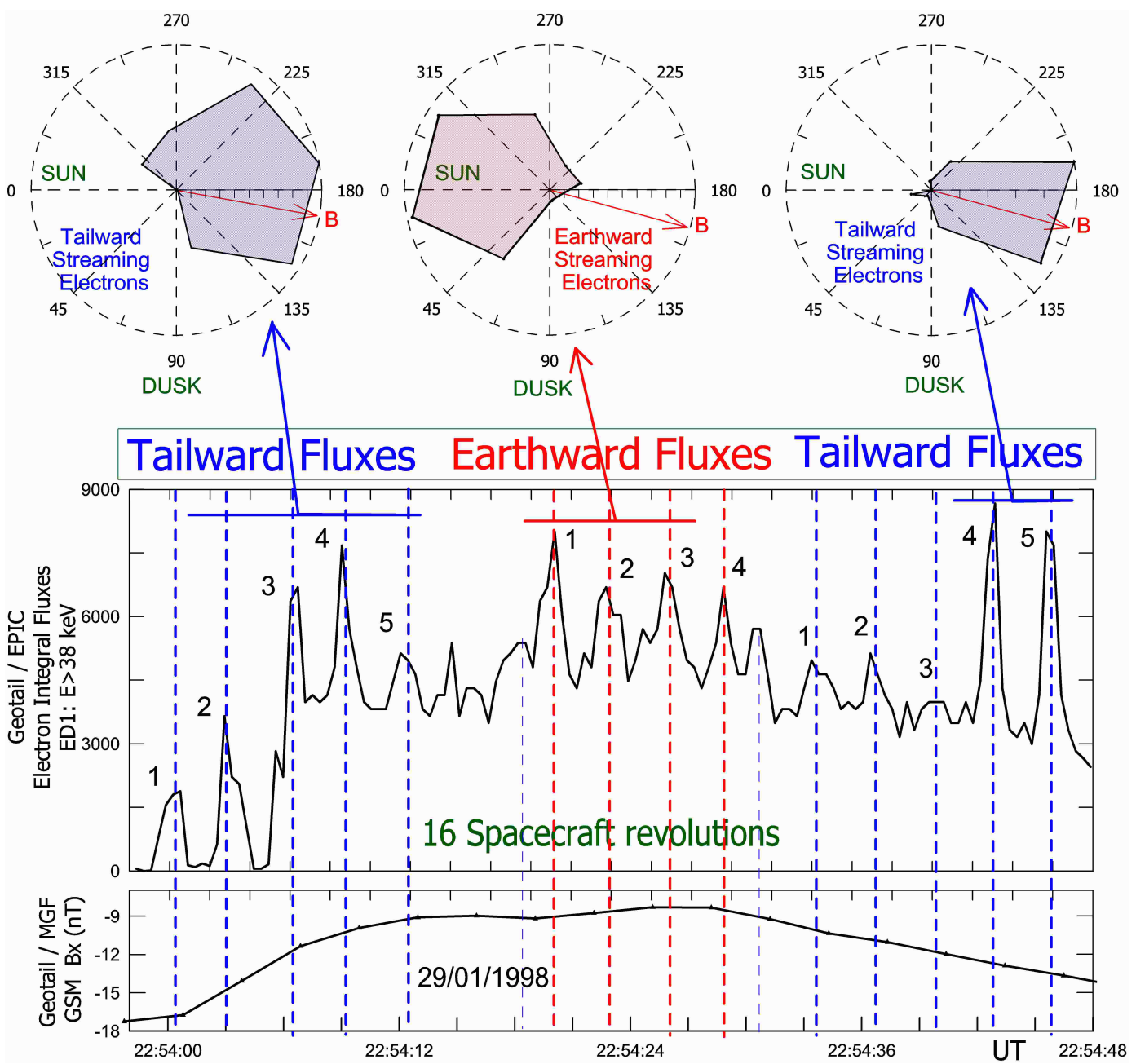

Fig. 3. An electron bi-layer event observed within an interval of 50-s; almost the same format as in Fig. 1. The satellite performs a short excursion departing from lobes to the deeper-central plasma sheet, while the electron fluxes change from tailward to earthward and from earthward to tailward in close succession.

\subsection{Event 5}

This event (Fig. 5) resembles the already shown, except that the under study interval is much longer (i.e., $11.66 \mathrm{~min}$ ). From 05:27:15 to 05:29:50 UT (i.e., between the blue-dashed vertical demarcation lines), we observe an uninterrupted tailward flow of energetic electrons. At times, an earthward directed population also coexists; however, the tailward fluxes dominate. That is, for $\sim 2.5 \mathrm{~min}$ (i.e., $\sim 50$ satellite revolutions), we observe persistent and highly anisotropic tailward electron fluxes. An indicative angular distribution being the overall response for the interval 05:28:14-05:28:27 UT (i.e., for 4 spacecraft revolutions) is shown at the lower insert; the average magnetic field direction is also displayed. Apparently the electrons stream tailward, and the tailward to earthward flux ratio, at times, exceeds the value of 30 . After this interval, a few more cycles with tailward streaming electrons are detected around 05:32:45 UT, whereas the first earthward streaming electrons are detected at $\sim 05: 34: 35 \mathrm{UT}$. A long-lasting interval of $\sim 42$ s (i.e., 14 spacecraft revolutions) with uninterrupted earthward electron fluxes is observed around 05:36 UT. An indicative angular distribution made up of data collected from 5 sequential spacecraft revolutions, from 05:36:08 to 05:36:24 UT, is shown at the upper insert; the electrons actually stream earthward and along the magnetic field lines. It is worth noticing that the layer with tailward streaming electrons is essentially confined in a region adjacent to the lobes; it is associated with high $\left|\boldsymbol{B}_{\mathrm{x}}\right|$ values. The layer with earthward streaming electrons is found in the central plasma sheet region.

\subsection{Event 6}

This event (Fig. 6) resembles better to the third one showing a brief inward satellite excursion into the plasma sheet; it is observed on 25 January 1998. Initially Geotail moves 

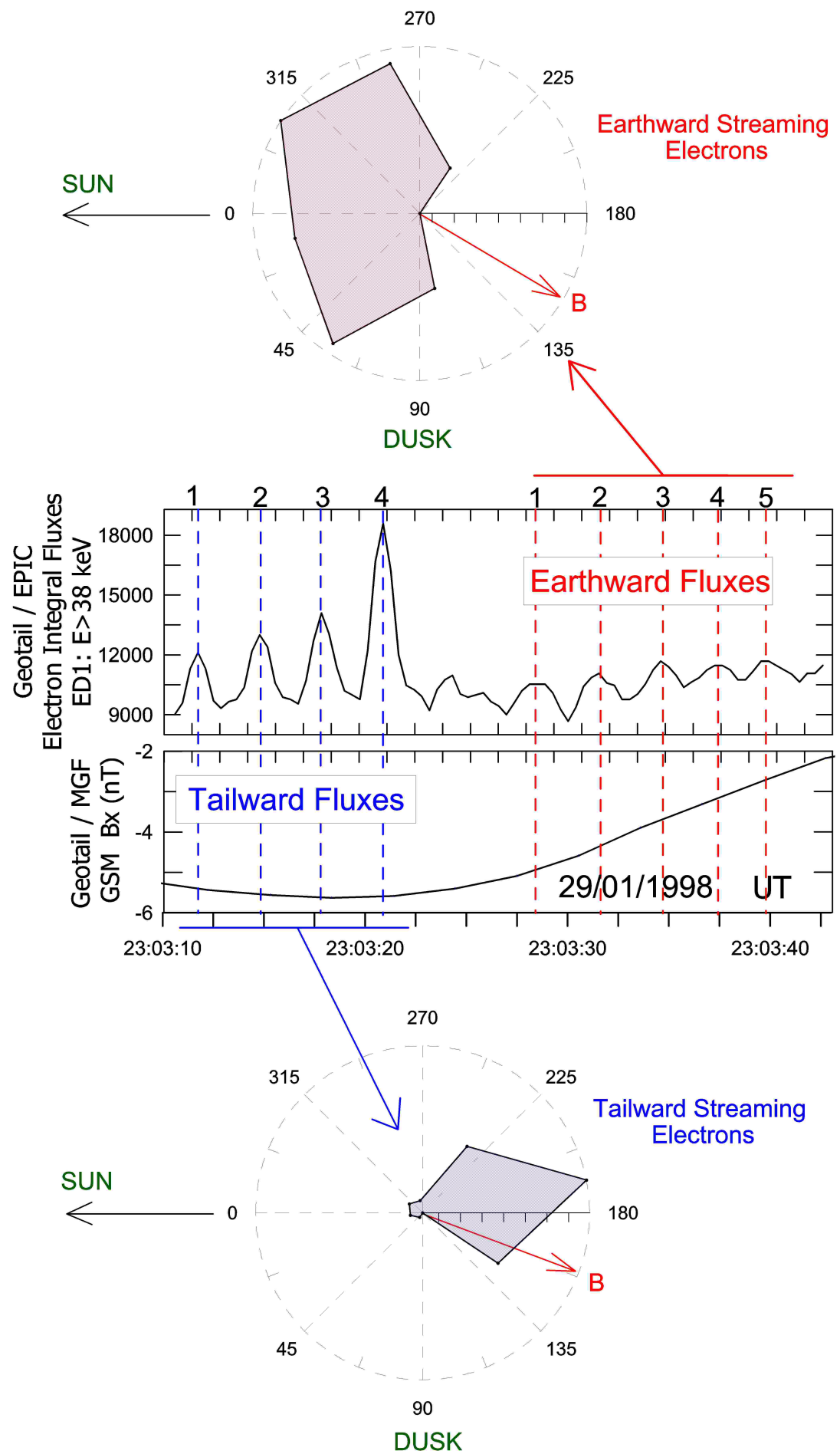

Fig. 4. A 33-s interval (i.e., 10 satellite revolutions) showing a very smooth transition from tailward to earthward electron fluxes; same format as in Fig. 1.

slightly inward and later switchbacks to its departure point in the plasma sheet. The brief $\boldsymbol{B}_{\mathrm{X}}$ excursion corresponds to a fluctuation $\Delta \boldsymbol{B}_{\mathrm{x}} \approx 2 \mathrm{nT}$; its duration is less than $2 \mathrm{~min}$. The energetic electron fluxes change successively and in close as- sociation with the $\boldsymbol{B}_{\mathrm{x}}$ variations: The earthward fluxes occur deeper in the plasma sheet; in contrast, the tailward fluxes occur at higher $\left|\boldsymbol{B}_{\mathrm{x}}\right|$ values. The three indicatively shown angular distributions (together with the projected vector magnetic 


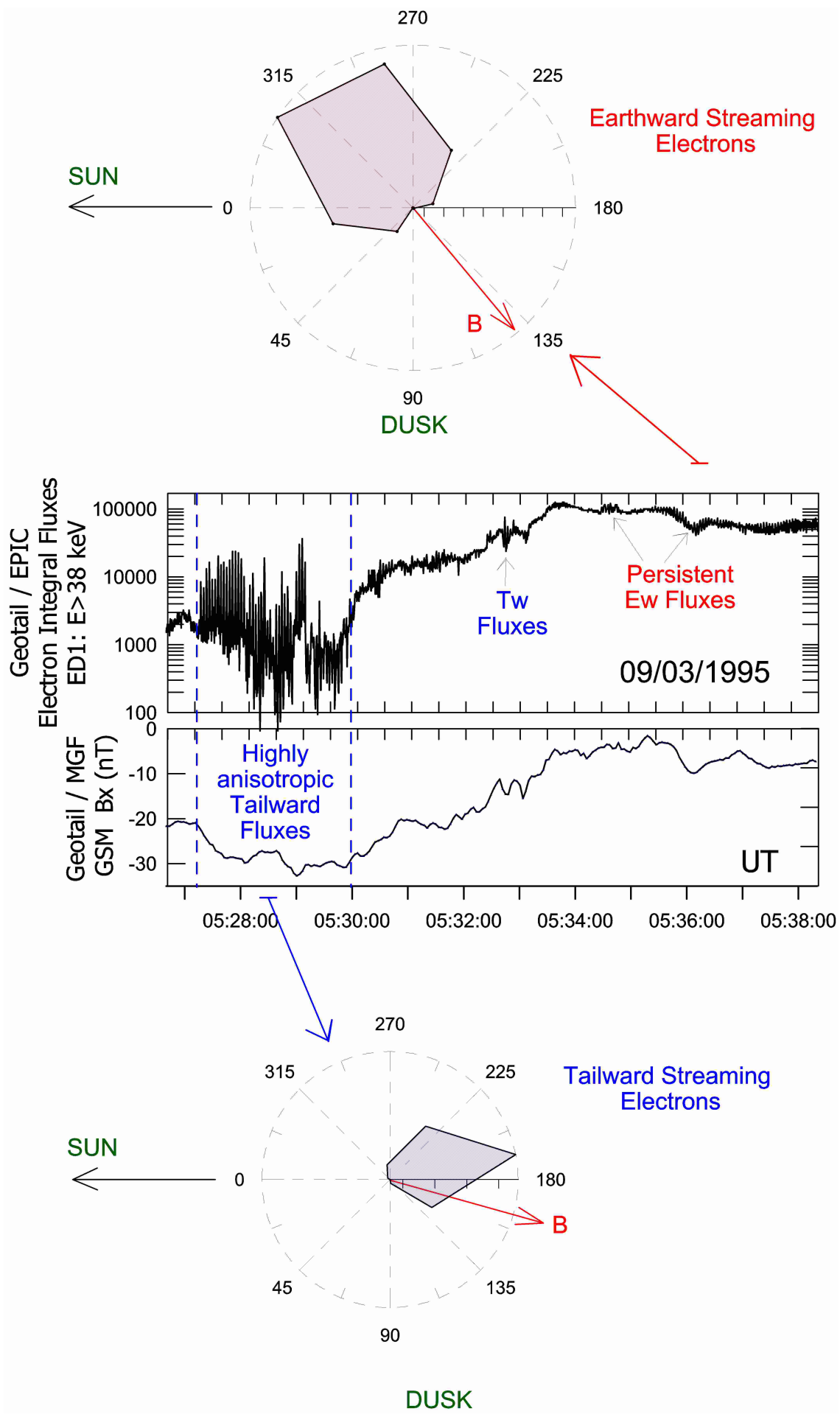

Fig. 5. Highly anisotropic tailward electron fluxes observed close to the plasma sheet boundary layer are switched to earthward in the central plasma sheet. Two indicative angular distributions are also shown.

field over the XY plane) emphasize the alternate electron flows. Therefore, we identify a bi-layer spatial structure with anti-parallel streaming energetic electrons. The inner layer is characterized by earthward fluxes and the outer layer by tailward fluxes. 


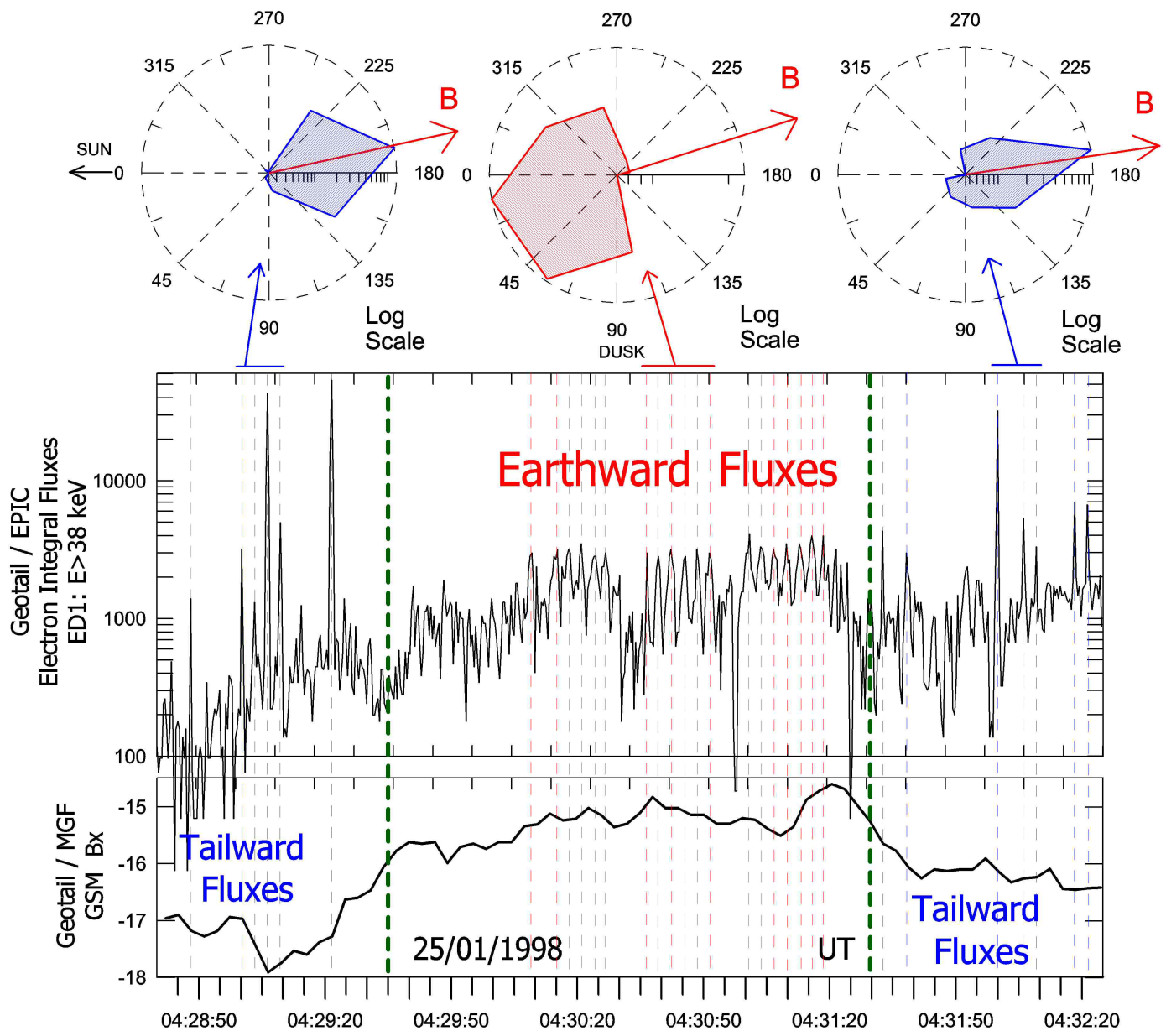

Fig. 6. Initially Geotail moves slightly inward and later switchbacks to its departure point in the plasma sheet. The brief $\boldsymbol{B}_{\mathrm{X}}$ excursion corresponds to a fluctuation of $\Delta \boldsymbol{B}_{\mathrm{X}} \approx 2 \mathrm{nT}$ and the energetic electron fluxes change successively and in close association with the $\boldsymbol{B}_{\mathrm{X}}$ variations: The earthward fluxes occur deeper in the plasma sheet; the tailward fluxes occur at higher $\left|\boldsymbol{B}_{\mathrm{X}}\right|$ values. The three indicatively shown angular distributions (and the projected vector magnetic field over the XY plane) emphasize the changes in electron flows.

It is worth noticing that the earthward fluxes persist for $\sim 20$ satellite revolutions (i.e., $\sim 1 \mathrm{~min}$ ); each peaked flux (corresponding to one Geotail revolution) is marked by a reddashed vertical line. At times the tailward fluxes are high and extremely collimated along the ambient magnetic field lines; these beams probably constitute an electron population which is more effectively accelerated.

\section{$2.7 \quad$ Event 7}

This event occurs $\sim 5$ min later than the preceded one; both of them belong to the same substorm episode. It although covers a period of just $110 \mathrm{~s}$ (Fig. 7), however, the shortlived satellite penetration deeper into the plasma sheet is very clear. The inward motion (from $\boldsymbol{B}_{\mathrm{x}} \approx-8$ to $-5 \mathrm{nT}$ ) is associated with a distinct transition from tailward to earthward fluxes, whereas the outward motion (from $\boldsymbol{B}_{\mathrm{x}} \approx-5$ to $-8 \mathrm{nT}$ ) is associated with a flow transition in the reverse order. The bi-layer structure is further visualized showing three indicative angular distributions with the methodology used in the preceded examples. The duration for each angular distribution is marked with a horizontal bar; the reader can count the satellite revolutions (successive dashed-vertical lines) used in each case.

\section{Discussion}

The discussion is divided into three subsections: First, we interested in for the timing of the electron bi-layer events within their own substorm framework in terms of local plasma sheet thinning and local plasma sheet recovery. Second, we stress the fact that these events are identified for the first time and, therefore, it naturally follows that none single paper or researcher is focused on them in the past. Any electron acceleration mechanism proposed in the passed does not automatically can claim to produce the bi-layer feature. Third, we make our own suggestion for an electron 


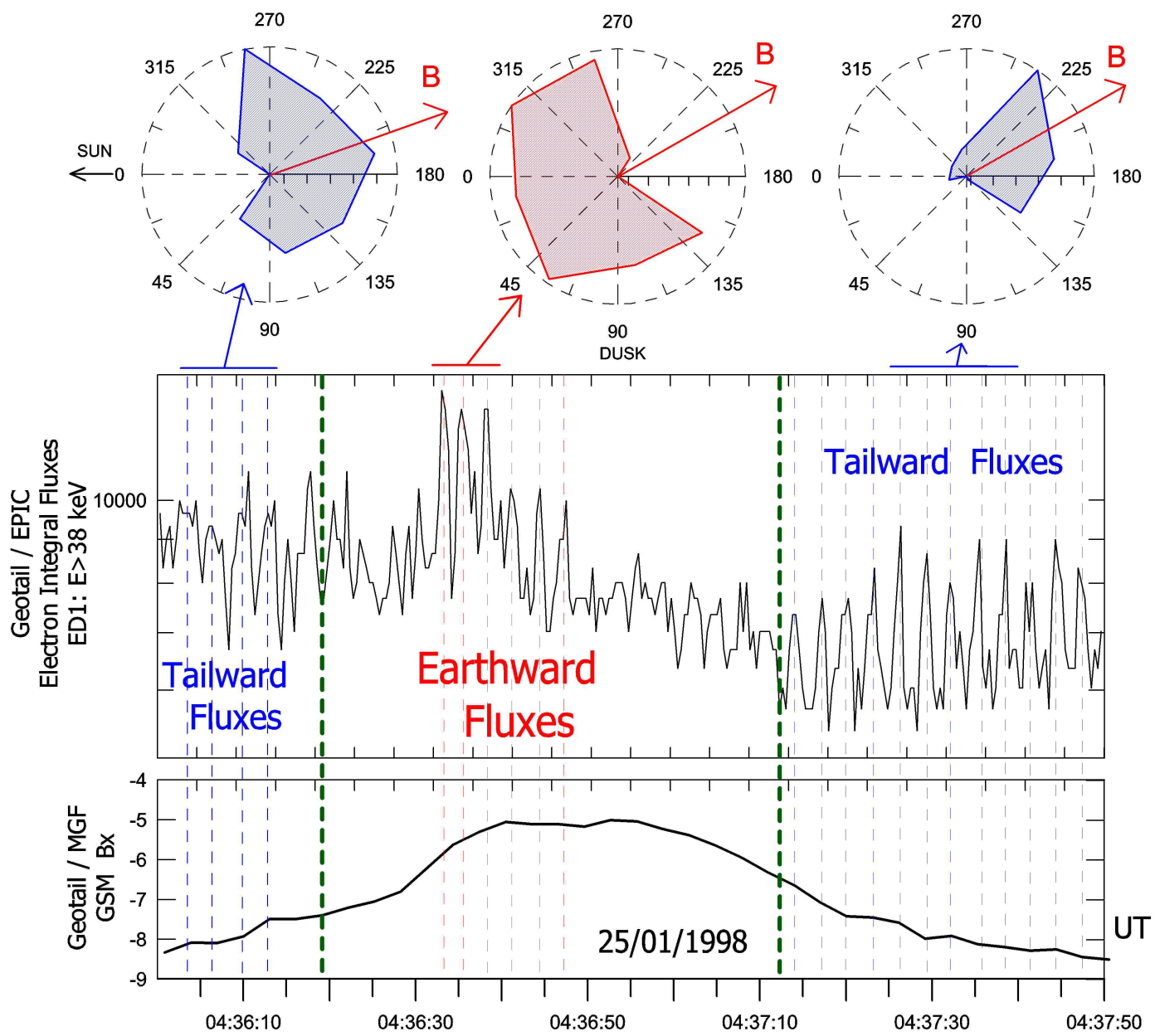

Fig. 7. This event occurs $\sim 5 \mathrm{~min}$ later than the preceded one and although covers a period of just $110 \mathrm{~s}$, however, the short-lived satellite penetration deeper in plasma sheet is very clear. The inward motion (from $\boldsymbol{B}_{\mathrm{X}} \approx-8$ to $-5 \mathrm{nT}$ ) is associated with a distinct transition from tailward to earthward fluxes, whereas the outward motion (from $\boldsymbol{B}_{\mathrm{X}} \approx-5$ to $-8 \mathrm{nT}$ ) is associated with a flow transition in the reverse order.

acceleration mechanism potentially producing all the observed characteristics.

\subsection{Occurrence site of bi-layer events}

We are particularly interested in determining the satellite position relatively to the active source site; a knowledge determining the basic magnetic field topology. Our result is that the bi-layer events are sited tailward as well as earthward relatively to a supposed X-line activation source. We use the near Earth neutral line (NENL) model (e.g., Baker et al., 1996) as the typical reconnection scheme associated with plasma flows and particle accelerations in magnetotail. In this way we put forward the fundamental scheme on which is based every result concerning the relative satellite/source positions. According to this scenario the reconnection site is initially developed close to the Earth and later retreats tailward. A satellite tailward of the source (i.e., roughly tailward of the locally thinned plasma sheet in which the X-line is de- veloped) will initially receive intense tailward particle fluxes and later intense earthward fluxes. This simplified picture is sketched in Fig. 8, although here the satellite is set to move earthward in a stationary magnetic field configuration. Bellow, we scrutinize all the cases although in the first place, we discuss the more compelling ones. Since in this work four substorms are involved, it is logical that in each case only those parameters that suffice to fulfill our purpose are presented. A more detailed multi-satellite, multi-instrument analysis will be out of the scope of this work.

\subsubsection{Bi-layer events "6" and "7" of day 25, 1998}

The sixth and seventh bi-layer electron events are observed during a substorm on 25 January (DOY=25), 1998. Their precise timing, as the substorm proceeds, will potentially help us to understand the involved acceleration mechanism. Geotail was located at $(\mathrm{X}, \mathrm{Y}, \mathrm{Z})=(-28.6,-7.3,-4) R_{\mathrm{E}}$ at 04:30 UT. 


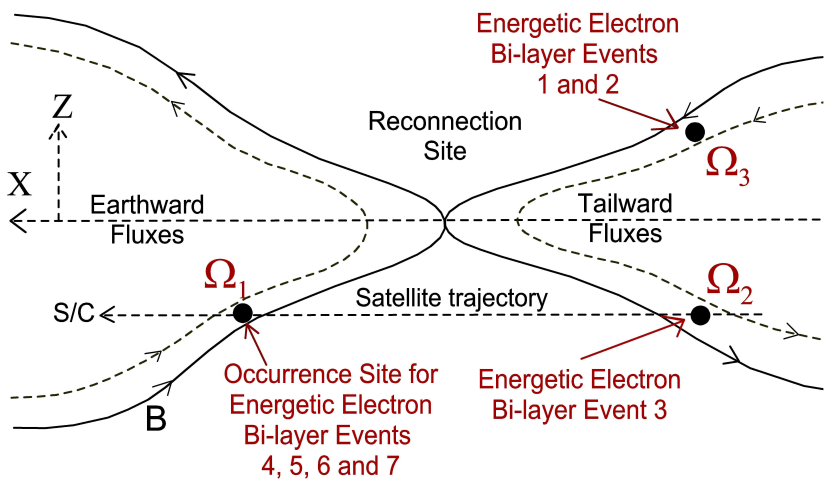

Fig. 8. A sketch for a typical X-type reconnection line with the substorm associated earthward and tailward plasma flows; the satellite $(\mathrm{S} / \mathrm{C})$ is considered propagating earthward in a stationary structure. The bi-layer events "4", "5", "6" and "7" occurred at the site $\Omega_{1}$, whereas the events " 1 ", "2", and " 3 " occurred at $\Omega_{2}$ and $\Omega_{3}$ at the right-hand side of the neutral line.

We look at Fig. 9 synthesized by only the necessary Geotail data-sets that suffice to prove our just articulated conclusion. From top to bottom, we plot (a) the $58-77 \mathrm{keV}$ proton differential fluxes; (b) the greater than $38 \mathrm{keV}$ integral electron fluxes; (c) the theta (polar) angle of the magnetic field; (d) the $V_{\mathrm{X}}$ component of the plasma velocity, and (e) the $\boldsymbol{B}_{\mathrm{X}}$ component of the magnetic field. Additionally, the most interesting interval of $\boldsymbol{B}_{\mathrm{X}}$ is shown in detail at the bottom panel trace: The satellite moves from the plasma sheet boundary region toward the neutral sheet and records the two bi-layer electron events marked with arrows. It is apparent that the just mentioned satellite motion is accompanied by weak earthward plasma fluxes, while a few minutes later the earthward plasma velocity exceeds the value of $1000 \mathrm{~km} \mathrm{~s}^{-1}$. Conversely, before this time the plasma flows were essentially tailward with a major measured velocity value $V_{\mathrm{x}} \approx-500 \mathrm{~km} \mathrm{~s}^{-1}$. The tailward plasma flows are associated with exceptionally intense tailward energetic proton fluxes, which are shown using the blue line at the top panel. These $58-77 \mathrm{keV}$ proton fluxes are switched to earthward when the plasma flows do the same. In addition, the theta angle trace of the magnetic field (third panel) in fact shows the local magnetotail depolarization process that is initiated at $\sim 04: 37 \mathrm{UT} ; \theta$ finally becomes $\sim 80^{\circ}$, a well established signature of the substorm's recovery phase. Therefore, we infer that the bi-layer electron events " 6 " and " 7 " are both detected just before the observed intense earthward fluxes; around the point of letter $\Omega_{1}$ in Fig. 8. This is an exceptional result; energetic electrons are observed to stream inward and outward of the "reconnection site" simultaneously.

It is worth noticing that the tailward electron fluxes are probably not reflected populations on Earth. In the opposite case we should observe the reverse order of flows in bi-layer events: Earthward fluxes at larger $\boldsymbol{B}_{\mathrm{x}}$ and tailward fluxes at slightly decreased $\boldsymbol{B}_{\mathrm{x}}$ values, given that the electrons are sub- jected to the $\boldsymbol{E}_{\mathrm{y}} \times \boldsymbol{B}_{\mathrm{x}}$ drift toward the central plasma sheet. However an extended discussion is undertaken later on.

\subsubsection{Bi-layer events “3” and " 4 " of day 29,1998}

In this paragraph we deal with the so-called " 3 " and "4" bilayer events, which are associated with a substorm on $29 \mathrm{Jan}$ uary 1998. Figure 10 is essentially synthesized with the same format as the Fig. 9; it is focused on the under study substorm episode, too. The intense tailward (earthward) fluxes for the 58-77 keV protons, in the top panel, along with the very high tailward (earthward) plasma flows, in the bottom panel, and the mostly negative (positive) theta angles of the magnetic field are manifestations of the fact that the satellite passes by an X-line as it is illustrated in Fig. 8. Thus, we infer that the bi-layer event " 3 " occurs close to the site $\Omega_{2}$ (tailward plasma flows) and the bi-layer event "4" occurs close to the site $\Omega_{1}$ (earthward plasma flows).

\subsubsection{Bi-layer events " 1 " and "2" of day 248,1996}

These events are also substorm associated and observed on 4 September 1996. From the large amount of datasets accompanying these events, we have indicatively selected the absolutely necessary parameters plotted in Fig. 11. Around 21:35 UT the negative theta angle of the magnetic field along with the (weak) tailward plasma flows close to neutral sheet $\left(\boldsymbol{B}_{\mathrm{X}}=0 \mathrm{nT}\right)$ assure us that the satellite was located within or very close to the acceleration source (reconnection site). 10 and $15 \mathrm{~min}$ later the bi-layer electron events " 1 " and " 2 " are observed, while (weak) tailward plasma flow continues to flow. Thus, we infer that both events occurred in north plasma sheet and tailward of an X-line; at the site $\Omega_{3}$, which is the symmetric of $\Omega_{2}$ with respect to the X-axis (Fig. 8).

\subsubsection{Bi-layer event " 5 " of day 68,1995}

The fifth bi-layer electron event is briefly commented as follows: First, the geostationary satellite 1990-095 shows at $\sim 05: 05$ UT an electron injection at $\mathrm{LT} \approx 02: 30$ (not shown here), and the under study electron event occurs $\sim 30 \mathrm{~min}$ after the injection time. In Fig. 5 persistent tailward electron fluxes are mainly observed with $\boldsymbol{B}_{\mathrm{x}} \approx-25 \mathrm{nT}$; the earthward electron fluxes are observed later with $\left|\boldsymbol{B}_{\mathrm{x}}\right|<5 \mathrm{nT}$. One can infer that the satellite gradually approaches the neutral sheet; at the same time the plasma velocity is slightly earthward $\left(\sim 100 \mathrm{~km} \mathrm{~s}^{-1}\right.$, not shown here) and the magnetic field recovers to its dipole-like configuration. Therefore, we deduce that bi-layer event " 5 " was located close to the site $\Omega_{1}$ in Fig. 8. 

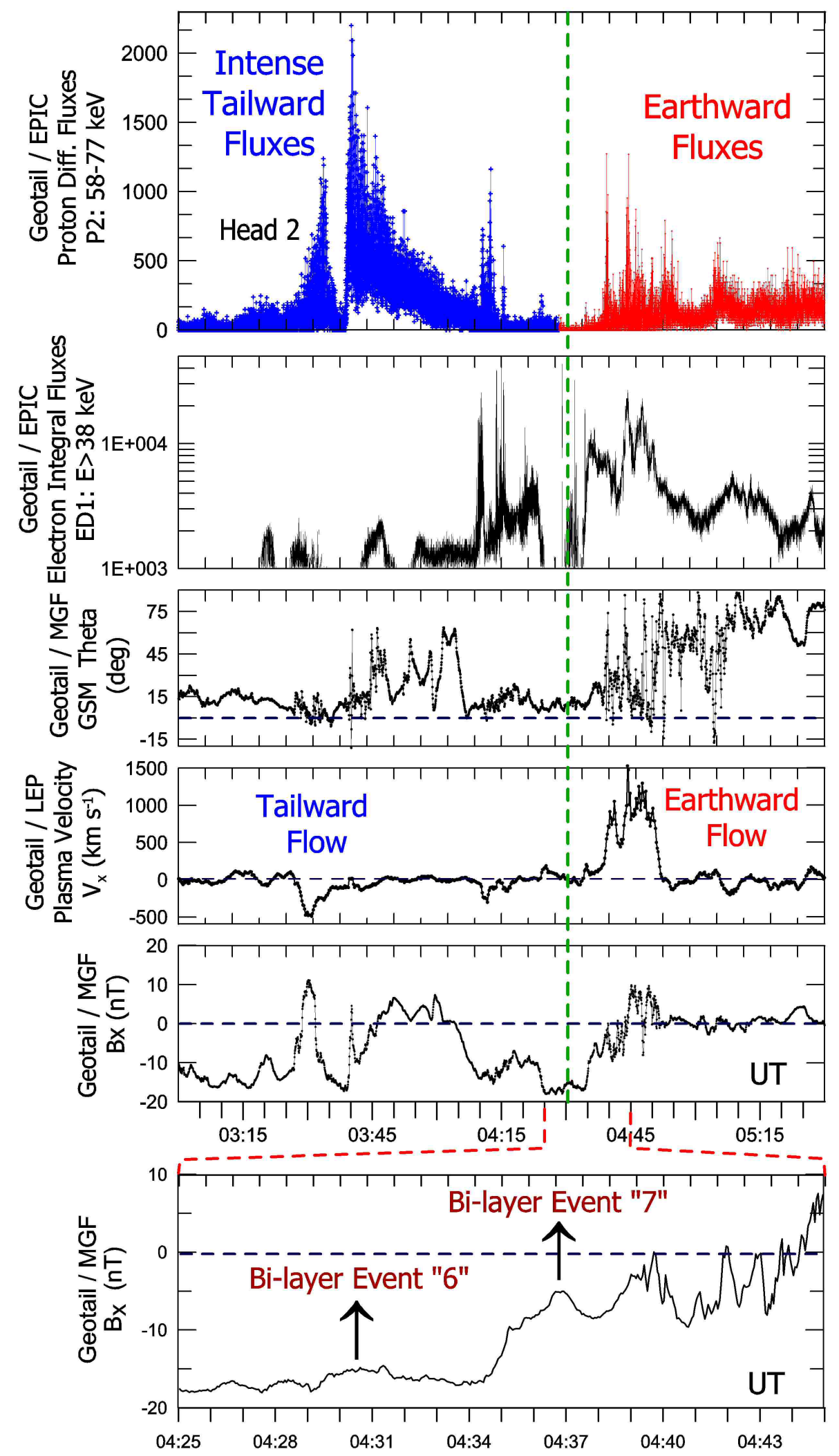

Fig. 9. Data-sets concerning an isolated substorm occurred on 4 September 1996. Geotail datasets from top to bottom: (a) Proton differential fluxes $(58-77 \mathrm{keV})$ and $(\mathbf{b})$ electron integral fluxes $(E \geq 38 \mathrm{keV})$ from the EPIC/ICS instrument, (c) theta angle of the magnetic field (in degrees), (d) $V_{\mathrm{X}}$ component of the plasma velocity (in $\mathrm{km} \mathrm{s}^{-1}$ ), (e) $\boldsymbol{B}_{\mathrm{X}}$ component of the magnetic field, and (f) a close up of $\boldsymbol{B}_{\mathrm{X}}$ concerning the interval 04:25-04:45 UT wherein the bi-layer events "6" and "7" are observed. 


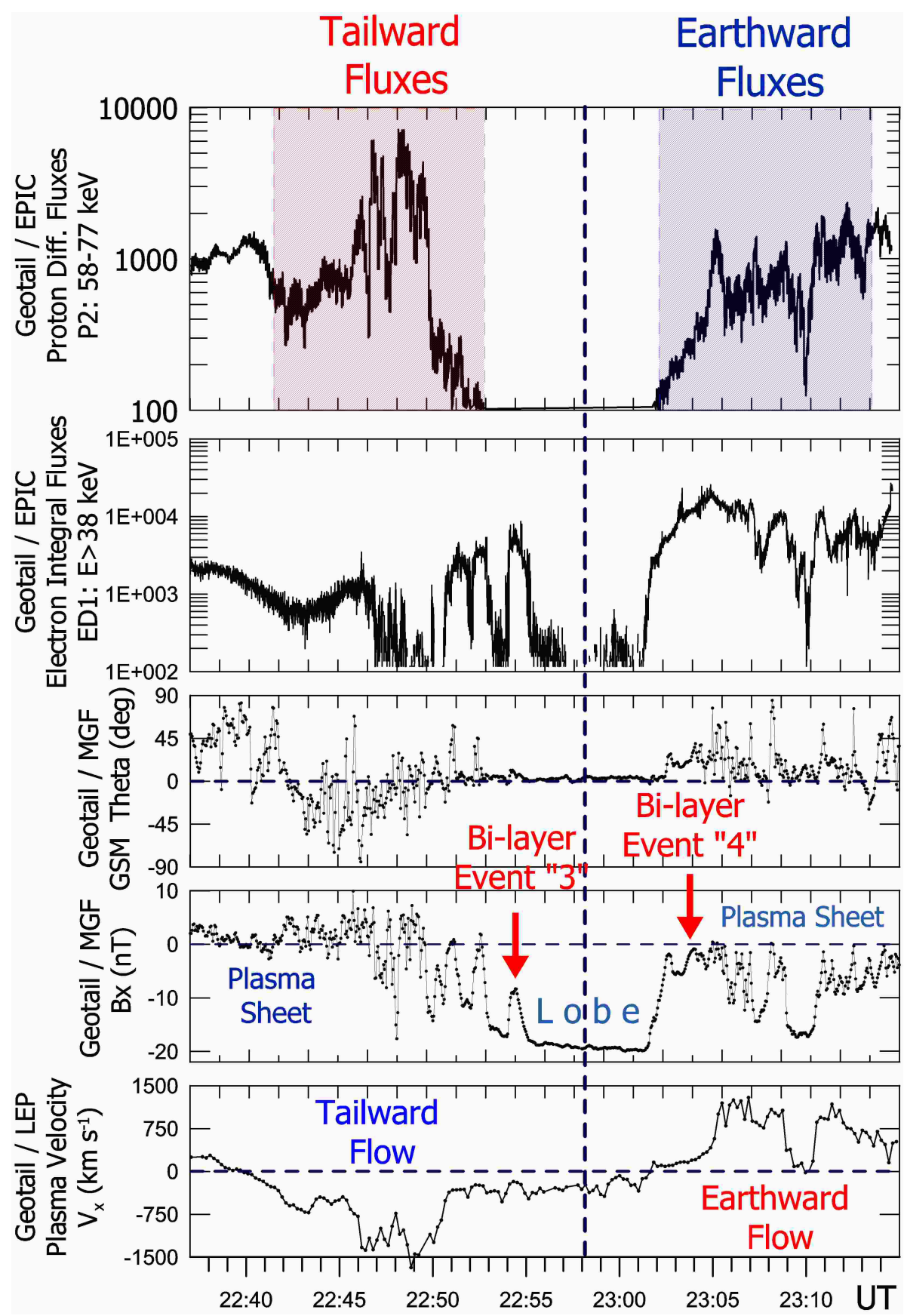

Fig. 10. Data-sets concerning the bi-layer events "3" and "4" occurred during the substorm on 29 January 1998. The tailward plasma flows are observed in parallel with the tailward energetic proton fluxes (red shaded area at the top panel), while the earthward plasma flows are observed in parallel with earthward energetic proton fluxes (blue shaded area at the top panel). The format is similar with that of Fig. 9.

\subsection{Three significant issues associated with the bi-layer events}

\subsubsection{Tailward streaming electrons, not reflected beams}

First and foremost we must discriminate between the tailward reflected electrons and those which are locally tailward accelerated. Actually, the earthward fast moving electrons can be mirrored near Earth by the converging magnetic field lines and be reflected tailward. Williams (1981), in his early ISEE-1 work, demonstrated that energetic ions streaming tailward along the $\boldsymbol{B}$ are simply the result of mirror point reflection of an earthward moving ion population. In the case of much more faster moving electrons, we anticipate 


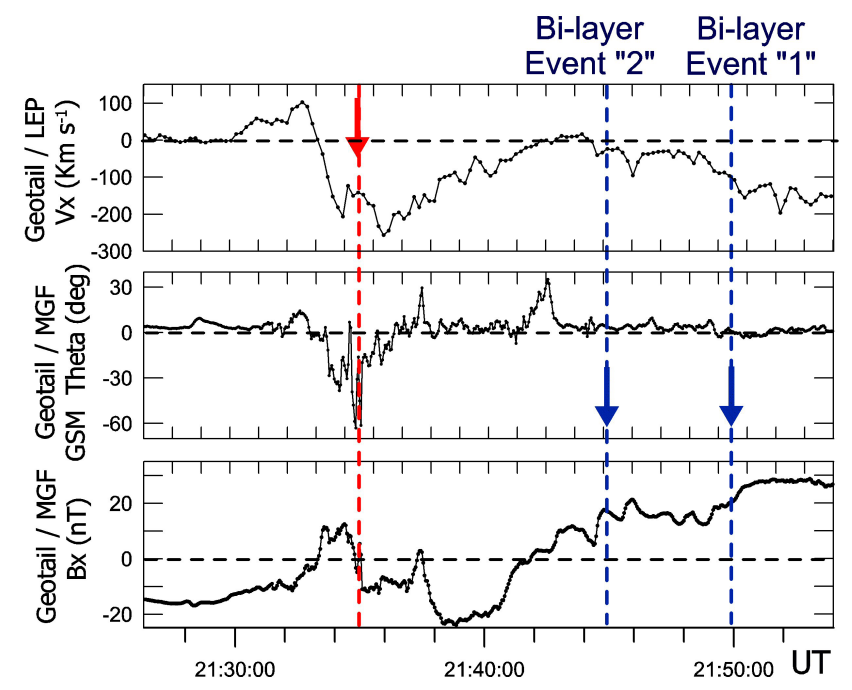

Fig. 11. Datasets for the $V_{\mathrm{x}}$ component of plasma velocity along with the theta angle and the $\boldsymbol{B}_{\mathrm{X}}$ component of the magnetic field associated with the bi-layer events " 1 " and " 2 ". We observe that both events occurred with slightly tailward plasma flow.

that almost immediately with the earthward electron fluxes, tailward ones must occur; hence, bi-directional fluxes like those studied by Shiokawa et al. (2003) should be finally observed. In contrast, we have observed, at least in Fig. 5, an $\sim 160$-s uninterrupted tailward electron flow. The round trip of reflected electrons for the under study events is $20-60 R_{\mathrm{E}}$, and the travel time does not exceed the 1-3s. Thus, if the tailward electrons are reflected on Earth, then we ought to observe rather bi-directional electron angular distributions, or (in scarce cases) the tailward electrons should be observed at lower $\boldsymbol{B}_{\mathrm{x}}$ values given that the electrons are affected by the $\boldsymbol{E}_{\mathrm{y}} \times \boldsymbol{B}_{\mathrm{x}}$ drift toward the neutral sheet. However, in all our cases (together with those observed just at the plasma sheet/lobe interface) the tailward streaming electrons have occurred on magnetic field lines characterized by higher $\boldsymbol{B}_{\mathrm{x}}$ values; a result of great importance. For instance, in Fig. 1 the tailward electron flow from 21:49:20 to 21:49:50 UT has occurred with $\boldsymbol{B}_{\mathrm{X}}$ greater than $18 \mathrm{nT}$; in Figs. 2 and 3 the tailward fluxes around 21:45:50 and 22:54:00 UT, respectively, occurred adjacent to the lobes where, as it is anticipated, the flux intensities sharply decrease. Similarly, in Fig. 5 around 05:29:00, and in Fig. 6 around 04:29:10 UT, tailward electron fluxes have apparently occurred in a lobe-like domain. Therefore, systematically the tailward streaming electrons are detected at the external plasma sheet layer (in cases adjacent to the lobes). Additionally, in all cases the pairs of parallel and anti-parallel layers are well-separated; a feature that is later interpreted adopting the notion of two distinct and spatially separated antiparallel electric fields.

\subsubsection{Electron acceleration mechanisms proposed in the past}

Apparently we do not reject the electron acceleration mechanisms proposed in the past; it is significant to remove any misconception associated with this issue. For instance, Hoshino et al. (2001) studied the origin of the hot and suprathermal electrons in terms of the kinetic magnetic reconnection process by using simulations. Additionally, Hoshino (2005) suggested the "electron surfing acceleration mechanism in magnetic reconnection" based on the polarization electric fields pointing toward the neutral sheet in a thin plasma sheet. In the akis geometry the Hoshino's electron surfing acceleration mechanism may work in a similar way as in the X-type magnetic reconnection configuration. Their V-shaped magnetic field geometry and the (cross-magnetic field) polarization electric fields are similar with the akis geometry and its associated orthogonal electric fields. The observed electron bi-layer structure may add something very important to their mechanism.

Moreover, Fujimoto and Machida (2006) simulated a generation mechanism of high-energy electrons in the plasma sheet/lobe boundary region associated with magnetic reconnection. All these efforts (plus more of them not cited here) are not questioned here; however, it is clear that these mechanisms do not automatically reproduce the observed electron bi-layer feature; nobody has predicted or discussed such a layered spatial structure of counterstreaming energetic electrons.

Hoshino (2005) in his simulation results produced an "electron plasma sheet boundary layer (PSBL) beam of hot electrons" flowing away the X-type region and, in the outer boundary region, a beam of cold electron flowing toward the $\mathrm{X}$-type region. His results are consistent with the observations of Nagai et al. (2001), who "commonly observed inflowing less than $5 \mathrm{keV}$ electrons in the outermost layer of the plasma sheet in magnetic reconnection events, where accelerated ions and electrons flow away from the magnetic reconnection region. Finally, the latter mentioned works are differentiated from our study in two elements: First, our distinct antiparallel beams are both composed of energetic electrons; they did not detect any energetic electron beam moving toward the X-line. Second, we use integral electron measurements greater than $38 \mathrm{keV}$, while they used $5 \mathrm{keV}$ electrons; hence, we can not discuss any result related to $5 \mathrm{keV}$ electrons, it does not add something to our study; its out of the field defined in the title of this work.

Fujimoto et al. (2001) reported on electrons, in the $0.1 \mathrm{keV}$ to a few $\mathrm{keV}$ energy range, that were seen to flow tailward at the lobe/plasma sheet interface in the near Earth $\left(X<-15 R_{\mathrm{E}}\right)$ magnetotail with their pitch angles highly collimated along the field lines. They proposed that the tailward electrons sustain the downward field aligned current (FAC) originating from the near Earth X-line magnetotail reconnection. Certainly we also observe tailward electron flows, 


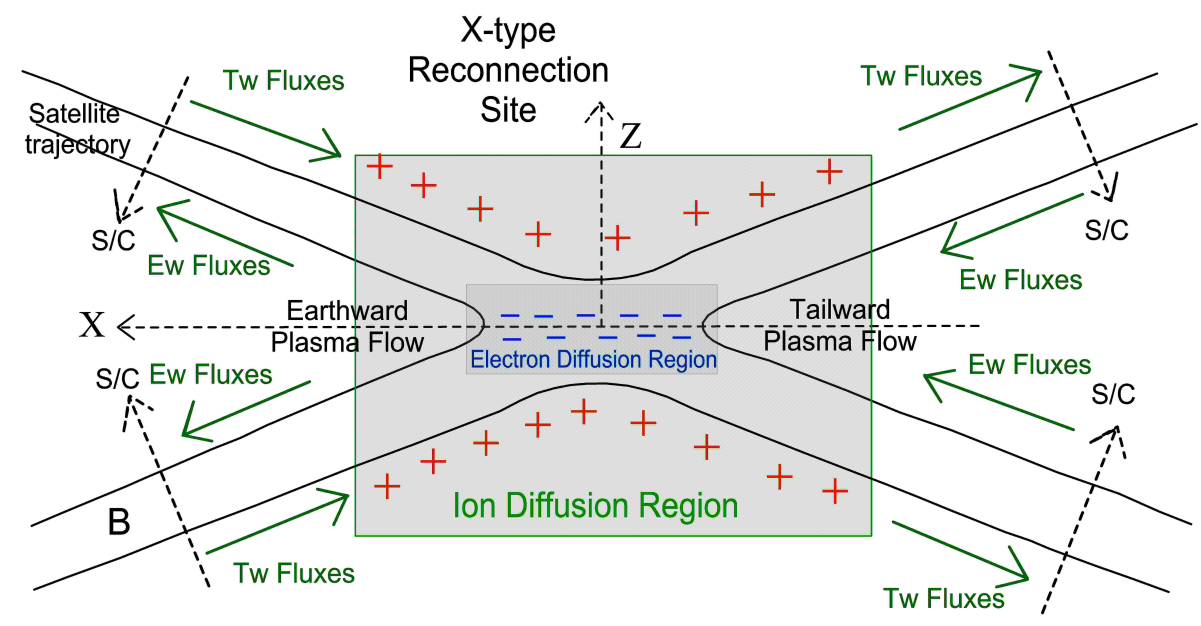

Fig. 12. Under the X-type reconnection model, as a satellite is crossing inward the separatrix, regardless of plasma flow direction, the electron bi-layer structure may be encountered. That is, we should always observe tailward (Tw) streaming electron fluxes in the layer closer to the lobes and earthward (Ew) electron fluxes in the layer closer to the central plasma sheet.

although with much more energetic electrons; most importantly, our selected events concern bi-layer structures with earthward electron fluxes, too. We, as well as Fujimoto et al. (2001), study streaming electrons, but beyond this common ground, we discuss essentially different issues.

\subsubsection{Brief discussion pertinent to the $\mathrm{X}$-line model}

The observed (Sarris et al., 1976) acceleration of plasma sheet particles to energies above the electrostatic potential across the magnetosphere is interpreted in terms of the tail reconnection that involves large inductive electric fields. Many authors (Coppi et al., 1966; Schindler, 1974; Galeev and Zelenyi, 1975; Galeev et al., 1978) have recognized that if the collisionless tearing mode is unstable in the tail and grows to large amplitudes, it could provide rapid dissipation of the tail magnetic energy and the inductive acceleration of energetic particles. Later Baker et al. (1979) presented an acceleration scheme based on the above cited works and the work of Sarris and Axford (1979); energetic particles stream outward from the X-line site and along the newly reconnected magnetic field lines. From these early works onward the Xline reconnection model, in general, is supposed to produce suprathermal ions and electrons. However, the studied bilayer electron events seem to dictate additional features on the acceleration source; they impose several constraints on the activated center. The micro-mechanism governing the Xline reconnection must generate and the bi-layer domain as well.

The studied layered structures pose a fundamental question. So far it is considered as a commonplace that energetic electrons stream always outward from an activated center, the $\mathrm{X}$-line; however, we observe outcoming and incoming energetic electrons simultaneously. Most importantly, the problem has an additional dimension resulted by the relative oc- currence positions between the bi-layer events and the supposed reconnection site, the $\mathrm{X}$-line. Figure 8 visualizes our result: Four bi-layer events observed earthward of the X-line and three events are observed tailward of it. Therefore, the big challenge for any candidate mechanism is its potentiality to produce all these observed features. It should be, again, stressed that to our Knowledge there is no a theory or a simulation effort predicting or discussing the bi-layer energetic electron structure.

Again, if we attempt to address the issue on the basis of the X-line model, then we should have a geometry like that sketched in Fig. 12. We consider that the typical X-line model is associated with an excess amount of demagnetized ions in the ion diffusion region and an excess amount of electrons in the electron diffusion region, so that between them a polarization electric field is directed to the neutral point in the inflow region. The candidate acceleration mechanism has to always produce tailward (Tw) electron fluxes closer to the lobes, and earthward (Ew) electron fluxes in the layer closer to the central plasma sheet. At the time being it should be clearly stated: We did not resolve the raised question; it was impossible to find a mechanism producing the electron bilayer structures on the basis of an X-type reconnection line. This is an open issue for future research efforts.

Nevertheless, we consider that it is legitimate to try an explanation scenario which is based on the "akis dynamics", as it is introduced by Sarafopoulos (2008). The akis structure seems to be much promising, with its own magnetic field topology and charge decoupling mechanism. In our consideration, there are significant similarities and differentiations, as far as akis is compared to the standard X-type theory. The identification of alternate mechanisms does not diminish the $\mathrm{X}$-line models as viable possibilities. Moreover, our purpose is not to contradict the $\mathrm{X}$-line reconnection model; our 


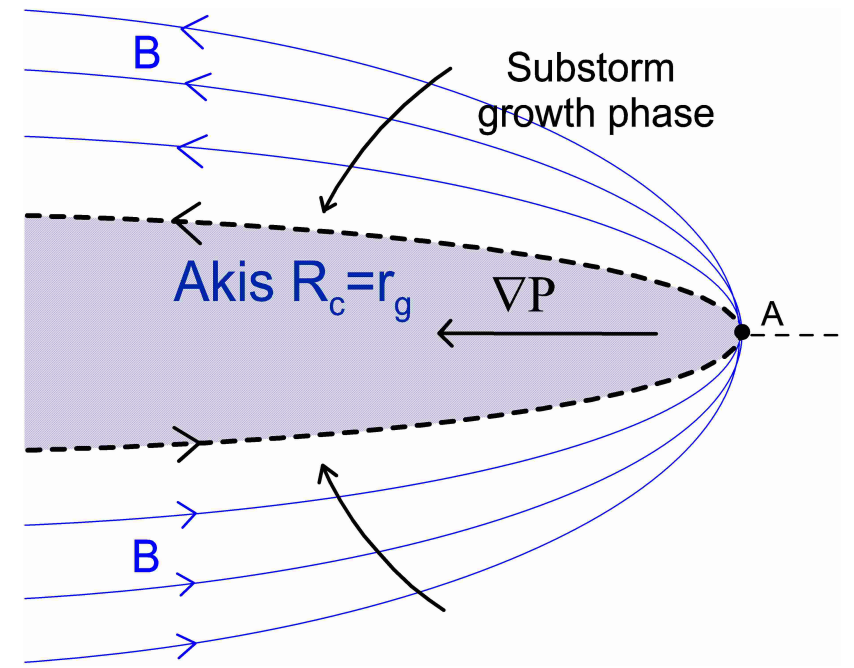

Fig. 13. Schematic illustrating the structure of plasma sheet close to the ion chaotization boundaries. During the substorm's growth phase the magnetotail magnetic field lines are stretched out from the Earth. At a Near Earth point A, when the magnetic field line satisfies the criterion of $R_{\mathrm{C}}=r_{\mathrm{g}}$ (thick-dashed line), then the ions are entirely pitch angle scattered and the cross-tail current will be exclusively depended on $\nabla_{\perp} P_{\perp}$.

motivation remains to scrutinize the properties of the akis geometry. The dynamics of akis may be the prime trigger that accelerates all the other processes in magnetotail. In this case, we will offer something related with the correct identification of processes in the cause-and-effect relationship, hence the physics.

As a result, this author undertakes an effort to suggest a mechanism that potentially produces the exhibited observations. Moreover, the latter leads to a micro-mechanism that potentially causes cross-tail current disruption; in this view, our proposed scheme would be considered as a complementary theory to the current disruption or magnetic reconnection ones (Lui et al., 2008). In essence, we suggest that the electric field formed in front of akis may be catalytically important to fire the tail magneto-plasma and trigger off the substorm episode. Eventually, we propose that the akis dynamics indeed may lead to current disruption and/or magnetic reconnection. Obviously, this work opens new conversation; unanswered questions remain and future work is necessary. This moment our main concern is that the proposed mechanism does not violate any basic magneto-plasma law. Our suggestion will be largely a qualitative approach; further thorough theoretical analysis and simulation efforts are needed.

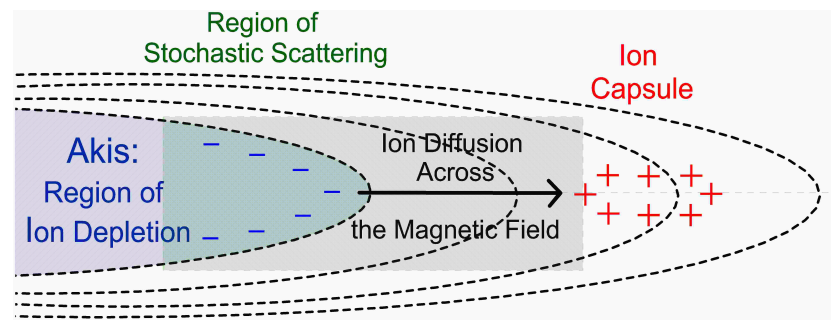

Fig. 14. The akis is formed when the chaotization process reaches its full-scale at $R_{\mathrm{C}}=r_{\mathrm{g}}$. Moreover, the akis ions will be stochastically scattered by strong wave turbulence and under the influence of $\nabla_{\perp} P_{\perp}$ will be diffused tailward transversely to the magnetic field lines; this way the ion capsule region with net positive charge is formed.

\subsection{An approach based on the akis dynamics}

\subsubsection{Magnetic field topology related to akis}

It is of particular interest to briefly introduce-identify the three well-separated regions in the near Earth magnetotail related to the akis structure. These regions are as follows:

Region 1: This region termed "akis" by Sarafopoulos (2008) loses a portion of its hot ion content; the tailward ejected population is composed of demagnetized ions. The prime pre-requisite leading to the development of akis is the plasma sheet thinning process near the Earth during the growth phase of a substorm. The magnetic field steadily obtains a more stretched geometry, like that shown in Fig. 13; a result well understood from early works (e.g., look at Fig. 3 in the work of Schindler, 1974). Furthermore, we assume that the critical configuration of akis is set by the limit $R_{\mathrm{c}}=r_{\mathrm{g}}$ (where $R_{\mathrm{c}}$ and $r_{\mathrm{g}}$ are the local magnetic field curvature and ion gyroradius, respectively; see the Introduction section). In the near Earth plasma sheet, while the substorm associated local thinning process is underway, the pitch-angle scattering process of ions becomes significant when $R_{\mathrm{c}} \leq 9 r_{\mathrm{g}}$. The ions are led to an isotropization when $R_{\mathrm{c}}=r_{\mathrm{g}}$. Additionally, the non-adiabatic ions of akis interact with strong wave turbulence and therefore they undergo a transition to stochastic scattering. These demagnetized ions of akis diffuse tailward across the magnetic field. This way the akis region behind the boundary $R_{\mathrm{c}}=r_{\mathrm{g}}$ is characterized by an excess of negative charge, as it is shown in Fig. 14. Certainly an outflow of ionospheric ions should be motivated to neutralize the negative charge of akis in the plasma sheet. However, the latter is a very slow process that needs one to two hours (Cladis, 1886; Delcourt et al., 1999; Chappell et al. 2000).

Region 2: The akis is encapsulated by an electric field $\boldsymbol{E}_{\perp}$, which is directed inward (earthward) and perpendicular to the local magnetic field lines on the flanks of akis. This orthogonal electric field in many ways resembles with the polarization electric field in the inflow region of an X-type reconnection configuration. 


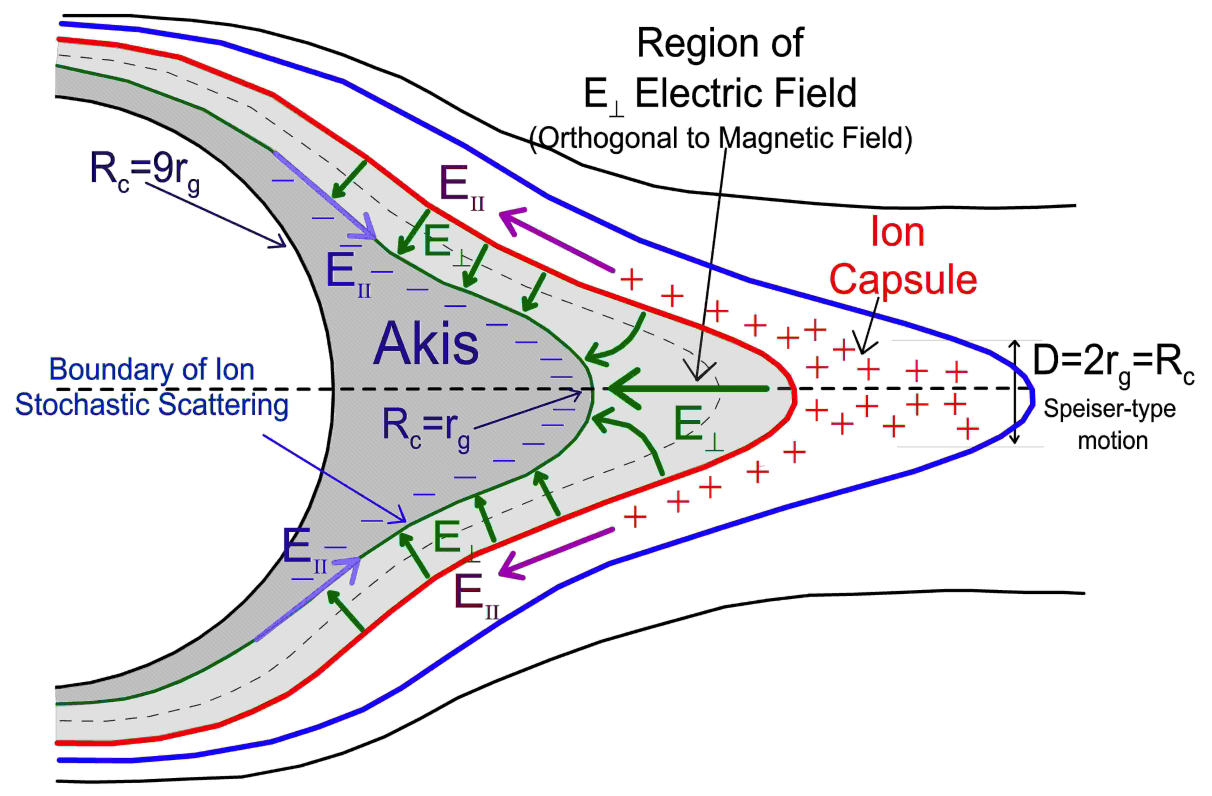

Fig. 15. In a near Earth thinned plasma sheet three distinct regions may be embedded: The akis region, the region of orthogonal $\boldsymbol{E}_{\perp}$ electric field and the ion capsule region. Tailward of akis (ion donor) is formed the capsule region (ion receptor). Akis is negatively charged and an electric field $\boldsymbol{E}_{\perp}$ perpendicular to the magnetic field lines is developed in the space between akis and capsule. The akis topology supports charge separation and magnetic-field aligned $\boldsymbol{E}_{\|}$electric fields which potentially drive field-aligned currents and accelerate electrons.

Region 3: Tailward of the region of electric field $\boldsymbol{E}_{\perp}$ another region is formed by the ion diffusion process: The so called "ion capsule region" characterized by a net positive charge. The configuration of the proposed ensemble of three regions is illustrated in Fig. 15. Certainly, somewhere in the ion capsule region with $R_{\mathrm{c}} \leq r_{\mathrm{g}} / 2$ the ions will presumably perform the Speiser-type motion (Speiser, 1965). It is apparent that, in general, the $R_{\mathrm{c}}$ monotonically decreases at greater distances from the Earth, something which is impressively shown by the Pulkkinen et al. (1992) simulations. Below our fundamental assumptions are more clearly cited.

The ensemble of regions of akis, $\boldsymbol{E}_{\perp}$ and capsule develops phenomena very similar to those taking place at the junction of a p-n diode. Akis is the ion donor and capsule is the ion acceptor; the diffusion is at the core of processes. If the region in between akis and capsule becomes depleted of charge carriers, then it will behave as an insulator; $\boldsymbol{E}_{\perp}$ is the electric field that is developed through the depletion zone-region having a depletion width.

It is meaningful to mention two more works that essentially are based in a stretched topology (like that of akis) for the near Earth magnetotail. Liu (2001) developed a formulation generating bursty bulk flows (BBFs) of plasma without introducing any NENL model; instead he based on "a highly curved current sheet". Additionally, Wanliss et al. (2002) using a test particle model were able to produce fast tailward plasma flows in the near Earth central plasma sheet region, as a consequence of plasma sheet boundary layer ion beam dynamics. Both of the just cited works are exclusively fo- cused on the substorm growth phase. Magnetic field models like that of Wanliss et al. (2000) potentially are valuable tools approaching the "akis" dynamics.

\subsubsection{Fundamental assumptions}

Fundamental assumptions concerning the akis structure, its properties and its key role are as follows:

First assumption: In a stationary plasma with anisotropic pressures $P_{\perp}$ and $P_{\|}$(where, the subscripts $\perp$ and II refer to perpendicular and parallel to the magnetic field, respectively), the perpendicular total current $j_{\perp}$ (Baumjohann and Treumann, 1996) is given by

$j_{\perp}=\frac{\nabla_{\perp} P_{\perp}}{B}+\frac{P_{\|}-P_{\perp}}{B R_{\mathrm{c}}}$

Inside the ion chaotization boundary $\left(R_{\mathrm{c}}=r_{\mathrm{g}}\right)$ the ions are considered pitch angle scattered and the pressure is isotropic $\left(P_{I I}=P_{\perp}\right)$, which eliminates the current contribution from "the curvature term". Therefore, the total magnetic force (Fig. 13) is

$F=\nabla_{\perp} P_{\perp}$

Second assumption: We already considered that as the $R_{\mathrm{c}}$ curvature tends to become equal of $r_{\mathrm{g}}$, the ions become nonadiabatic. At the limit $R_{\mathrm{c}}=r_{\mathrm{g}}$, we assume that the demagnetization process is also under way and the ions do not move with the magnetic field lines. Moreover, the frozen-in magnetic field condition is violated (by strong wave turbulence) 
and consequently, "plasma elements which are at one time in a common magnetic field line may be on different field lines at another time" (Fälthammar, 2004). In this way, an ion diffusion region is formed in the akis region much like that formed around an X-type line in a thin plasma sheet. We can argue that the motion of particles in the strongly inhomogeneous magnetic field (of akis), as well as the interaction of particles with waves, lead to stochastic scattering of the particles (e.g., Büchner and Zelenyi, 1989; Chen, 1992).

Third assumption: Since the ion particles of akis are stochastically scattered and an intense pressure gradient is present at the same time, then we can describe our situation in terms of anomalous diffusion: Ion diffusion takes place across the magnetic field; that is, ions are ejected tailward of akis. The concept of ion diffusion is also discussed by a few researchers (e.g., Swift, 1992; especially by Lyatsky, 1999, and references therein). This way an "ion capsule" or an ion pocket region is formed. Field-aligned currents will be initiated to neutralize the newly formed net positive charge.

Fourth assumption. During the substorm's growth phase the magnetotail magnetic field lines are stretched out from the Earth (Sergeev et al., 1996; Rostoker, 1996; Baker et al., 1996; Lui, 1996). As the substorm's growth phase is underway new magnetic field lines will satisfy the criterion of $R_{\mathrm{c}}=r_{\mathrm{g}}$ and a tailward ejected demagnetized ion population will always exist. The ensemble of akis-capsule can be a long-lived structure. The situation is similar to that of the inflow region in the $\mathrm{X}$-type reconnection geometry. In our scenario the electrons in the akis neighbourhood remain adiabatic.

Fifth assumption. If we presume an impulsive (almost instantaneous) ion diffusion process, then the life-time for the positive capsule charge would be no more than $\sim 30 \mathrm{~s}$. The cold ionosphere electrons moving to neutralize the net positive charge would arrive in the ion pocket region in 10-30 s; an arbitrary value deduced from its supposed role, as we shall see bellow. However, this time is very small if the akis dynamics is to be connected with the duration of local current disruption in near earth magnetotail, which is typically short and ranges from $\sim 1$ to $5 \mathrm{~min}$ according to Lui et al. (1992). In addition, it is worth noticing that, for instance, the detected time-life of burst-like tailward $\sim 60 \mathrm{keV}$ proton fluxes, for the day 29, 1998, substorm event (look at our Fig. 10), is $\sim 4$ min. Under these circumstances we make a speculative suggestion arguing that an additional mechanism is at work: as cold ionosphere electrons are travelling along the magnetic field lines to neutralize the ion charge, they are actually accelerated. Consequently, the electrons achieve their maximum kinetic energy and velocity when they cross the ion pocket region; an oscillatory motion would be established. If the electron bounce motion is repeated, for instance for 10 cycles, then the time-life for the pocket charge will be 100-300 s, that is comparable with the current disruption duration. As a matter of fact quasi-periodic variations along the north-south component of $\boldsymbol{B}$ are observed and discussed (e.g., Takahashi et al., 1987; Sarafopoulos, 2008). Their periodicities of $T \approx 20$-s are dictated, as we suppose, by the neutralizing electrons (FACs) of the ion capsule charge. Further treatment and elaboration of this mechanism, in association with in situ observations, will be our future research issue.

Sixth assumption: The magnetic field line curvature radii steadily decrease further away from the Earth. The akis structure is apparently not characterized by the minimum $R_{\mathrm{c}}$ in the magnetotail; certainly the minimum $R_{\mathrm{c}}$ may even allow the electron chaotization. We again stress that the akis is just associated with the full chaotization of ion orbits. Thus throughout all the three regions shown in Fig. 15, the electrons are assumed moving adiabatically. Pulkkimen et al. (1992) have modeled the Earth's magnetotail magnetic field during the substorm growth phase and clearly discriminated and computed the two distinct earthward boundaries of chaotization: The first for the thermal $(5 \mathrm{keV})$ ions tailward of $\sim 8 R_{\mathrm{E}}$, and the second for thermal ( $1 \mathrm{keV}$ ) electrons tailward of $\sim 12 R_{\mathrm{E}}$; for strong substorms the electron chaotization boundary is shown to be displaced closer to the Earth. The critical condition for the tearing mode instability derived by Büchner and Zelenyi (1987) may be reached in a small region inside the electron chaotic region (Pulkkimen et al., 1992).

Seventh assumption: The akis is not a homogeneously charged region with net negative charge; charge redistribution takes place along the magnetic field lines and leads to increased electron densities just at the tip region of akis (Sarafopoulos, 2008). Electrostatic forces push the electrons toward the tip where the charge density increases; the tip is working much like as in charged metallic conductors.

Eighth assumption: The plasma sheet thinning during the growth phase of a substorm proceeds gradually earthward (Sauvaud and Winckler, 1980; Pulkkinen et al., 1992). Therefore, at a given distance from the Earth the $R_{\mathrm{c}}$ continuously decreases; when $R_{\mathrm{c}}=r_{\mathrm{g}}$, the ejected ions tailward of akis supply with ions the capsule region. The latter condition may be satisfied close to the Earth, for instance, from 7 to $15 R_{\mathrm{E}}$ away from Earth.

\subsubsection{Magnetic-field aligned electric fields and layered electron events}

According to our scenario, inside the akis and ion capsule regions, the ideal MHD approximation breaks down; the frozen-in condition for the magnetic field lines is violated. Within the akis region the supposed strong wave turbulence leads to ion demagnetization and tailward diffusion; the frozen-in field condition is violated, and hence field-aligned electric fields are allowed to exist under such conditions (Fälthammar, 2004, and references therein). In contrast, in collisionless plasmas the magnetic-field aligned electric fields cannot exist, because the unimpeded motion of electrons and ions along magnetic field lines would "shortcircuit" them. 

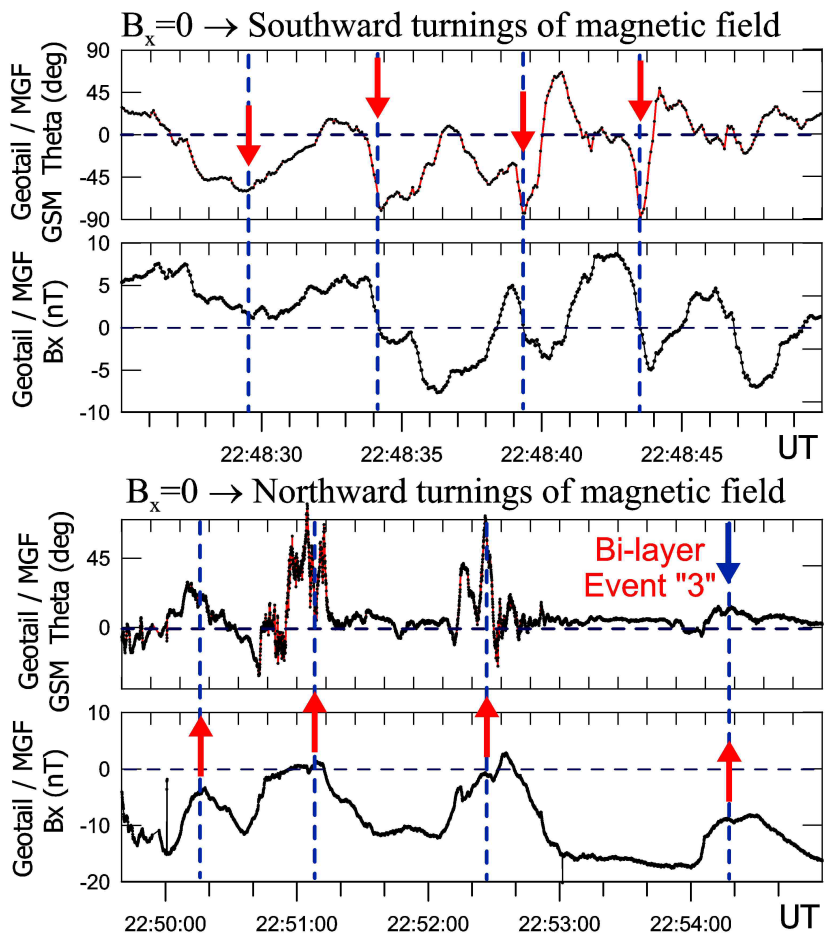

Fig. 16. Two intervals (almost in succession) showing two radically different plasma regimes in magnetotail. The upper two traces (being the first interval) show that, at times, the neutral sheet crossings are associated with intense southward turnings of the magnetic field. The bottom two traces show that each satellite approach to the neutral sheet is essentially accompanied by positive theta angles. Therefore, during the second interval (wherein the bi-layer event " 3 " occurred) the local magnetotail recovers to its "background geometry".

The net positive charge distributed within the capsule region produces an electric field $\boldsymbol{E}_{\|}$parallel to the magnetic field lines. This electric field accelerates the nearby electrons which move tailward neutralizing the net positive charge. Cold ionospheric electrons are also forced to be accelerated, all the way from the ionosphere to neutral sheet, since an $\boldsymbol{E}_{\|}$always exists along the magnetic field line given that $\nabla \cdot \boldsymbol{E}_{\|}=\rho_{\text {total }} / \varepsilon_{o}$, where $\rho_{\text {total }}$ is the electron density. Therefore, tailward streaming electrons along the magnetic field lines could be produced; and they are indeed observed in bilayer events.

Similarly, the akis is already considered as a region where negative charge is trapped (Sarafopoulos, 2008). Hence, the akis may support a magnetic-field aligned $\boldsymbol{E}_{\|}$electric field which is directed almost tailward; electrons could be accelerated earthward, and actually they are detected in bilayer events. Consequently, the different polarity net charges (and the associated magnetic-field aligned $\boldsymbol{E}_{\|}$electric fields shown in Fig. 15 with blue and purple colours) result in the bi-layer electron structure. The antiparallels $\boldsymbol{E}_{\|}$are well separated in space; the tailward streaming electrons must always be observed closer to the lobes.

\subsubsection{Is bi-layer event " 3 " characterized by the akis geometry?}

The bi-layer event "3" occurred with tailward plasma flow $\left(V_{\mathrm{x}} \approx-300 \mathrm{~km} \mathrm{~s}^{-1}\right)$, and it was located at the site $\Omega_{2}$ in Fig. 8; this is the reason we further scrutinize if actually the akis structure was really developed. Figure 16 emphatically demonstrates the magnetic field topology, when the event " 3 " occurred. We deliberately plot the magnetic field for two selected intervals: The upper two traces (being the first interval) show that, at times, the neutral sheet crossings $\left(\boldsymbol{B}_{\mathrm{X}}=0\right)$ are associated with intense southward turnings of the magnetic field. One may argue that about four "very small plasmoid-like" structures could be counted in the shown data. It seems that exactly at this time, when we observe extreme tailward plasma flows and the highest energetic proton fluxes, the satellite is at the very core of the acceleration source. In contrast, a few minutes later this scenery is radically changed for the second interval: The bottom two traces show that in this situation each satellite approach to the neutral sheet is essentially accompanied by positive theta angles. The latter provides strong evidence that this time the local magnetotail is recovered to its "background geometry" in which the bi-layer event " 3 " occurred; as a matter of fact we detect a magnetic field geometry which may be as that sketched in Fig. 15. Therefore, we infer that the plasma flow direction is not the dominant and absolute criterion; we argue that rather the geometry of the magnetic field configuration is of prime importance. Although it is not directly related to this work, we think that it is worth noticing that around 22:41:30 UT in Fig. 10, tailward plasma flows indeed occur with positive magnetic field polar angles in the very central plasma sheet. Finally, we underline that for the events " 1 " and " 2 " with tailward plasma flows there is not detectable any neutral sheet crossing.

\subsubsection{Akis and cross-tail current disruption mechanism}

The isotropic angular distribution of ions inside the region of akis (when $R_{\mathrm{c}} \approx r_{\mathrm{g}}$ ) will lead to enhanced ionospheric loss cone precipitation at the auroral latitudes (Lyons and Speiser, 1982). Thus, ions close to being field-aligned will escape outside the akis region. Another fraction of hot demagnetized and stochastically scattered ions will be ejected into the ion capsule region establishing the orthogonal electric field $\boldsymbol{E}_{\perp}$ in the space in front of akis. Therefore, a possible mechanisms triggering cross-tail current disruption may be as follows: Once the orthogonal $\boldsymbol{E}_{\perp}$ is established, then newly diffused ions from akis would be rather subjected to a dawnward $\boldsymbol{E}_{\mathrm{x}} \times \boldsymbol{B}_{\mathrm{z}}$ drift. In addition, diamagnetic electrons continue to drift dawnward, too. The latter may be the key mechanism capable of producing cross tail current disruption. Instead, the cross-tail current increases in the capsule region. 


\subsubsection{Balance equation across a stretched magnetic field geometry}

We can obtain a more quantitative statement from the basic MHD equations perpendicular to the curved magnetic field lines in a stretched tail-like geometry. Hence, we write the equation of motion, for the late growth phase and just prior to the substorm onset, in the stationary case

$\nabla P_{\mathrm{x}}+\nabla\left(\frac{B_{\mathrm{z}}^{2}}{2 \mu_{0}}\right)=\hat{\boldsymbol{x}} \frac{B_{\mathrm{z}}^{2}}{\mu_{0} R_{\mathrm{c}}}$

where $\nabla P_{\mathrm{x}}$ represents tailward directed pressure gradient forces, and $\nabla P_{\mathrm{x}}=\boldsymbol{J}_{\mathrm{y}} \times \boldsymbol{B}_{\mathrm{Z}}$ represents electromagnetic forces. The term on the right-hand side corresponds to the magnetic tension effect and the second term (on the left-hand side) corresponds to the magnetic pressure effect. We may use this relation as far as strong wave turbulence is not added in the stretched geometry. Certainly any such approach is merely indicative in the akis region since the MHD approximation is considered to break down. Thus, we can hypothesize that the current disruption in front of akis probably increases the earthward directed force of akis magnetic tension. Consequently, the balance is locally disturbed and a certain magnetic flux tube associated with the tailward section of akis is forced to return to its initial dipole-like structure. This collapse will naturally produce a transient earthward convection surge, while the near Earth magnetic field lines pile up. A new akis will be developed slightly tailward and/or dawnward (or duskward) of the preceded one.

\section{Epilogue}

In this work we identify a promising bi-layer structure of counterstreaming energetic electron fluxes in the Earth's magnetotail, and establish its spatial character. This structure is observed regardless of any plasma flow direction (earthward or tailward). Apart from any interpretation scheme or candidate electron acceleration mechanism, we think that the observational part of this work is, by itself, a much promising result; a deeper understanding is obviously needed.

The electron layered structures introduce a new diagnostic tool for the electron acceleration mechanism. First, they impose new constraints on the $\mathrm{X}$-line reconnection producing energetic electrons. Second, they are studied in terms of the "akis model", as it is recently introduced by Sarafopoulos (2008). Akis potentially constitutes a huge reservoir from which the ion population is largely channeled tailward to the "ion capsule regions". We assume that net negative charge is trapped at the equatorial part of akis, and net positive charge is trapped inside the capsule region; these charges, which remain unshielded for a while, can allow magneticfield aligned electric fields to exist, since the ideal MHD approximation breaks down. Consequently these electrostatic charges can support two magnetic-field aligned $\boldsymbol{E}_{\|}$electric fields which finally produce the bi-layer electron events. Additionally, it is assumed that the akis is encapsulated by an electric field which is directed earthward and perpendicular $\left(\boldsymbol{E}_{\perp}\right)$ to the local magnetic field lines on the flanks of akis. We argue that the $\boldsymbol{E}_{\perp}$ can even cause cross tail current disruption in front of akis. The region of $\boldsymbol{E}_{\perp}$ is very similar to the depleted zone of charge carriers in a p-n diode junction.

Acknowledgements. Concerning the use of the highest resolution Geotail/EPIC data-sets, we are grateful to (a) A. T. Y. Lui, R. W. McEntire, E. T. Sarris, and D. J. Williams, (b) S. R. Nylund for his pre-processing of the data, and (c) The Johns Hopkins University/Applied Physics Laboratory that maintains the available data web site. Also we thank S. Kokubun and T. Mukai for the highresolution Geotail/MGF magnetic field and Geotail/LEP plasma data, respectively. Regarding the use of LANL energetic particle data, we thank Richard Belian and Geoffrey D. Reeves.

Topical Editor I. A. Daglis thanks two anonymous referees for their help in evaluating this paper.

\section{References}

Baker, D. N., Belian, R., Higbie, P., and Hones Jr., E.: High-Energy Magnetospheric Protons and Their Dependence on Geomagnetic and Interplanetary Conditions, J. Geophys. Res., 84(A12), 71387154, 1979.

Baker, D. N., Pulkkinen, T. I., Angelopoulos, V., Baumjohann, W., and McPherron, R. L.: Neutral line model of substorms: Past results and present view, J. Geophys. Res., 101, 12975-13010, 1996.

Baumjohann, W. and Treumann, R. A.: Basic Space plasma physics, Imperial College Press-ICP, 122-124, 1996.

Belian, R. D., Gisler, G. R., Cayton, T., and Christensen, R.: High$\mathrm{Z}$ energetic particles at geostationary orbit during the great Solar Proton Event Series of October 1989, J. Geophys. Res. 97, 16897-16906, 1992.

Büchner, J. and Zelenyi, L.: Chaotization of the Electron Motion as the Cause of an Internal Magnetotail Instability and Substorm Onset, J. Geophys. Res., 92(A12), 13456-13466, 1987.

Büchner, J. and Zelenyi, L.: Regular and Chaotic Charged Particle Motion in Magnetotail-like Field Reversals, 1. Basic Theory of Trapped Motion, J. Geophys. Res., 94(A9), 11821-11842, 1989.

Chappell, C. R., Giles, B. L., Moore, T. E., Delcourt, D. C., Craven, P. D., and Chandler, M. O.: The adequacy of the ionospheric source in supplying magnetospheric plasma, J. Atmos. Solar Terr. Phys., 62, 421-436, 2000.

Chen, J.: Nonlinear Dynamics of Charged Particles in the Magnetotail, J. Geophys. Res., 97(A10), 15011-15050, 1992.

Cladis, J. B.: Parallel acceleration and transport of ions from polar ionosphere to plasma sheet, Geophys. Res. Lett., 13, 893-896, 1986.

Coppi, B., Laval, G., and Pellat, R.: A model for the influence of the earth magnetic tail on geomagnetic phenomena, Phys. Rev. Lett., 16, 1207-1210, 1966.

Delcourt, D., Dubouloz, N., Sauvaud, J.-A., and Malingre, M.: On the origin of sporadic keV ion injections observed by InterballAuroral during the expansion phase of a substorm, J. Geophys. Res., 104(A11), 24929-24937, 1999. 
Fälthammar, C.-G.: Magnetic-field aligned electric fields in collisionless space plasmas - a brief review, Geofísica Internacional, 43(2), 225-239, 2004.

Fujimoto, M., Nagai, T., Yokokawa, N., Yamade, Y., Mukai, T., Saito, Y., and Kokubun, S.: Tailward electrons at the lobe-plasma sheet interface detected upon dipolarizations, J. Geophys. Res., 106(A10), 21255-21262, 2001.

Fujimoto, K. and Machida, S.: A generation mechanism of electrostatic waves and subsequent electron heating in the plasma sheetlobe boundary region during magnetic reconnection, J. Geophys. Res., 111, A09216, doi:10.1029/2005JA011542, 2006.

Galeev, A. A. and Zelenyi, L. M.: Metastable states of diffuse neutral sheet and the substorm explosive phase, Sov. Phys. J.E.T.P. Letters, 22, 170-172, 1975 .

Galeev, A., Coroniti, F., and Ashour-Abdalla, M.: Explosive Tearing Mode Reconnection in the Magnetospheric Tail, Geophys. Res. Lett., 5(8), 707-710, 1978.

Hoshino, M., Mukai, T., Terasawa, T., and Shinohara, I.: Suprathermal electron acceleration in magnetic reconnection, J. Geophys. Res., 106(A11), 25979-25997, 2001.

Hoshino, M.: Electron surfing acceleration in magnetic reconnection, J. Geophys. Res., 110, A10215, doi:10.1029/2005JA011229, 2005.

Imhof, W.: Fine Resolution Measurements of the L-Dependent Energy Threshold for Isotropy at the Trapping Boundary, J. Geophys. Res., 93(A9), 9743-9752, 1988.

Kokubun, S., Yamamoto, T., Acuna, M. H., Hayashi, K., Shiokawa, K., and Kawano, H.: The GEOTAIL magnetic field experiment, J. Geomagnetism and Geoelectricity, 46, 7-21, 1994.

Krimigis, S. M. and Sarris, E. T.: Energetic particle bursts in the Earth's magnetotail, in: Dynamics of the Magnetosphere, edited by: Akasofu, S. I., Reidel Publishing Co., 599-630, 1980.

Liu, W. W.: Bursty bulk flows without a near-Earth neutral line: Generation of fast intermittent flow in a highly curved magnetic field, J. Geophys. Res., 106, 289-300, 2001.

Lopez, R. E. and Baker, D. N.: Evidence for particle acceleration during magnetospheric substorms, The astrophysical Journal supplement series, 90, 531-539, 1994.

Lopez, R. E., Lui, A. T. Y., Sibeck, D. G., Takahashi, K., McEntire, R. W., Zanetti, L. J., and Krimigis, S. M.: On the relationship between the energetic particle flux morphology and the change in the magnetic field magnitude during substorms, J. Geophys. Res., 94, 17101-17119, 1989.

Lui, A., Lopez, R., Anderson, B., Takahashi, K., Zanetti, L., McEntire, R., Potemra, T., Klumpar, D., Greene, E., and Strangeway, R.: Current Disruptions in the Near-Earth Neutral Sheet Region, J. Geophys. Res., 97(A2), 1461-1480, 1992.

Lui, A. T. Y.: Current disruption in the Earth's magnetosphere: Observations and models, J. Geophys. Res., 101, 13067-13088, 1996.

Lui, A. T. Y., Angelopoulos, V., LeContel, O., Frey, H., Donovan, E., Sibeck, D. G., Liu, W., Auster, H. U., Larson, D, Li, X., Nosé, M., and Fillingim, M. O.: Determination of the substorm initiation region from a major conjunction interval of THEMIS satellites, J. Geophys. Res., 113, A00C04, doi:10.1029/2008JA013424, 2008.

Lyatsky, W.: A possible role of ion demagnetization in substorm generation, J. Geophys. Res., 104(A9), 19905-19910, 1999.

Lyons, L. and Speiser, T.: Evidence for Current Sheet Acceleration in the Geomagnetic Tail, J. Geophys. Res., 87(A4), 2276-2286, 1982.

Mitchell, D. G., Williams, D. J., Huang, C. Y., Frank, L. A., and Russell, C. T.: Current carriers in the near-earth cross-tail current sheet during substorm growth phase, Geophys. Res. Lett., 17, 583-586, 1990.

Mukai, T., Machida, S., Satio, Y., Hirahara, M., Terasawa, T., Kaya, N., Obara, T., Ejiri, M., and Nishida, A.: The low energy particle (LEP) experiment onboard the Geotail satellite, J. Geomagn. Geoelectr., 46, 669-692, 1994.

Nagai, T., Shinohara, I., Fujimoto, M., Hoshino, M., Saito, Y., Machida, S., and Mukai, T.: Geotail observations of the Hall current system; Evidence of magnetic reconnection in the magnetotail, J. Geophys. Res., 106, 25929-25949, 2001

Pulkkinen, T., Baker, D., Pellinen, R., Büchner, J., Koskinen, H., Lopez, R., Dyson, R., and Frank, L.: Particle Scattering and Current Sheet Stability in the Geomagnetic Tail During the Substorm Growth Phase, J. Geophys. Res., 97(A12), 19283-19297, 1992.

Rostoker, G.: Phenomenology and physics of magnetospheric substorms, J. Geophys. Res., 101, 12955-12973, 1996.

Sarafopoulos, D. V., Sarris, E. T., and Pavlos, G. P.: Estimate of the amplitude of transient electric fields in the magnetotail, Proceeding Conf. Achievements of the IMS (International Magnetospheric Study), 26-28 June 1984, Graz, Austria, ESA SP-217, 177-180, 1984.

Sarafopoulos, D. V. and Sarris, E. T.: Transient Field-Aligned Electric Fields inside the Plasma Sheet inferred from measurements of Energetic Particles, Planet. Space Sci., 35, 991-995, 1987.

Sarafopoulos, D. V. and Sarris, E. T.: Inverse Velocity Dispersion of Energetic Particle bursts inside the Plasma Sheet, Planet. Space Sci., 36, 1181-1199, 1988.

Sarafopoulos, D. V., Sarris, E. T., Angelopoulos, V., Yamamoto, T., and Kokubun, S.: Spatial structure of the plasma sheet boundary layer at distances greater than $180 R_{\mathrm{E}}$ as derived from energetic particle measurements on GEOTAIL, Ann. Geophys., 15, 12461256,1997 , http://www.ann-geophys.net/15/1246/1997/.

Sarafopoulos, D. V., Sidiropoulos, N., Sarris, E., Lutsenko, V., and Kudela, K.: The dawn-dusk plasma sheet asymmetry of energetic particles: An Interball perspective, J. Geophys. Res., 106(A7), 13053-13065, 2001.

Sarafopoulos, D. V.: A physical mechanism producing suprathermal populations and initiating substorms in the Earth's magnetotail, Ann. Geophys., 26, 1617-1639, 2008, http://www.ann-geophys.net/26/1617/2008/.

Sarris, E. T., Krimigis, S. M., and Armstrong, T. P.: Observations of magnetospheric bursts of high protons and electrons at $\sim 35 R_{\mathrm{E}}$ with IMP-7, J. Geophys. Res., 81, 2341-2355, 1976.

Sarris, E. T. and Axford, W. I.: Energetic protons near the plasma sheet boundary, Nature, 277, 460-462, 1979.

Sauvaud, J.-A. and Winckler, J.: Dynamics of Plasma, Energetic Particles, and Fields Near Synchronous Orbit in the Night-time Sector During Magnetospheric Substorms, J. Geophys. Res., 85(A5), 2043-2056, 1980.

Schindler, K.: A theory of the substorm mechanism, J. Geophys. Res., 79, 2803-2810, 1974.

Sergeev, V. A. and Tsyganenko, N. A.: Energetic particle losses and trapping boundaries as deduced from calculations with a realistic magnetic field model, Planet. Space Sci., 30(10), 999-1006, 
1982.

Sergeev, V. A., Pulkkinen, T. I., and Pellinen, R. J.: Coupled-mode scenario for the magnetospheric dynamics, J. Geophys. Res., 101, 13047-13065, 1996.

Shiokawa, K., Baumjohann, W., and Paschmann, G.: Bi-directional electrons in the near-Earth plasma sheet, Ann. Geophys., 21, 1497-1507, 2003, http://www.ann-geophys.net/21/1497/2003/.

Speiser, T.: Particle Trajectories in Model Current Sheets 1. Analytical Solutions, J. Geophys. Res., 70(17), 4219-4226, 1965.

Swift, D.: Effects of Ion Demagnetization in the Plasma Sheet, J. Geophys. Res., 97(A11), 16803-16816, 1992.

Takahashi, K., Zanetti, L., Lopez, R., McEntire, R., Potemra, T., and Yumoto, K.: Disruption of the Magnetotail Current Sheet Observed by AMPTE/CCE, Geophys. Res. Lett., 14(10), 1019$1022,1987$.
Wanliss, J. A., Sydora, R. D., Rostoker, G., and Rankin, R.: Origin of some anisotropic tailward flows in the plasma sheet, Ann. Geophys., 20, 1559-1575, 2002, http://www.ann-geophys.net/20/1559/2002/.

Williams, D.: Energetic Ion Beams at the Edge of the Plasma Sheet: ISEE 1 Observations Plus A Simple Explanatory Model, J. Geophys. Res., 86(A7), 5507-5518, 1981.

Williams, D. J., McEntire, R. W., Schemm II, C., Lui, A. T. Y., Gloeckler, G., Christon, S. P., and Gliem, F.: Geotail energetic particles and ion composition instrument, J. Geomagn. Geoelectr., 46, 39-57, 1994. 